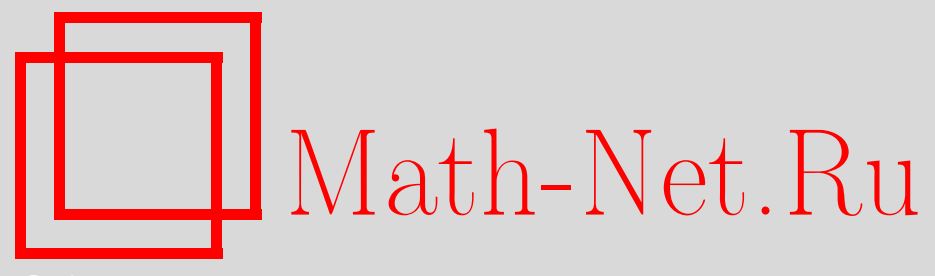

Г. И. Келбалиев, С. Р. Расулов, Математическое моделирование процессов коалесценции и дробления капель и пузырей в изотропном турбулентном потоке (обзор), Вестн. Сам. гос. техн. ун-та. Сер. Физ.-мат. науки, 2019, номер 3, 541-581

DOI: https://doi.org/10.14498/vsgtu1664

Использование Общероссийского математического портала MathNet.Ru подразумевает, что вы прочитали и согласны с пользовательским соглашением

http: //www.mathnet.ru/rus/agreement

Параметры загрузки:

IP : 3.80 .181 .102

26 апреля 2023 г., 10:18:54

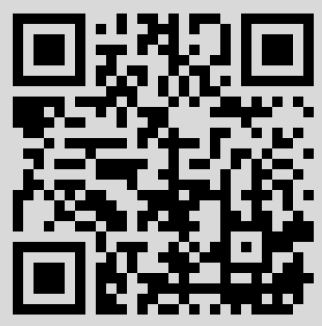


УДК 517.958:531.35

\title{
Математическое моделирование процессов коалесценции и дробления капель и пузырей в изотропном турбулентном потоке (обзор)
}

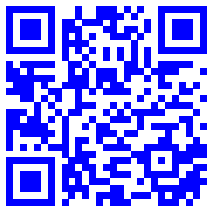

\author{
Г. И. Келбалиев ${ }^{1}$, С. Р. Расулов ${ }^{2}$ \\ 1 Институт катализа и неорганической химии НАН Азербайджана, \\ Азербайджан, AZ 1143, Баку, пр. Джавида, 29. \\ 2 Азербайджанский государственный университет нефти и промышленности, \\ Азербайджан, AZ 1010, Баку, пр. Азадлыг, 34.
}

\begin{abstract}
Аннотация
Предложенный обзор посвящен теоретическому анализу, расчету и моделированию процессов слияния и дробления капель и пузырей в изотропном турбулентном потоке. Проанализирован ряд исследований, посвященных этим проблемам. Рассмотрены вопросы определения минимальных и максимальных размеров капель и пузырей, а также частот дробления и слияния, которые связаны с решением диффузионного уравнения массопереноса. Слияние капель рассматривается как результат утончения межфазной пленки, образованной между двумя каплями в результате их столкновения. Предложено математическое описание утончения межфазной пленки с учетом эффекта Марангони. Анализ множества исследований, в том числе и собственных, показал, что в зависимости от масштаба турбулентных пульсаций экстремальный размер, а также частоты коалесценции и дробления капель и пузырей зависят от удельной энергии диссипации в турбулентном потоке, от их размеров и физических свойств частиц и среды. Важными параметрами, обеспечивающими агрегативную устойчивость дисперсной среды типа «жидкость - жидкость» или «жидкость - газ» к дроблению, деформации и слиянию, являются коэффициент поверхностного натяжения и диссипация энергии, физические свойства среды и частиц, а в изотропном турбулентном потоке - отношение коэффициента поверхностного натяжения к удельной энергии диссипации.

Рассмотрены также вопросы, связанные с эволюцией функции распределения частиц по времени и размерам в условиях изотропной турбулентности с использованием решений стохастического уравнения Фоккера-Планка для непрерывного изменения размеров капель и пузырей
\end{abstract}

\section{Обзор}

(2) (9) Контент публикуется на условиях лицензии Creative Commons Attribution 4.0 International (https://creativecommons.org/licenses/by/4.0/deed.ru)

\section{Образец для цитирования}

Келбалиев Г. И., Р асулов С. Р. Математическое моделирование процессов коалесценции и дробления капель и пузырей в изотропном турбулентном потоке (обзор) // Вестн. Сам. гос. техн. ун-та. Сер. Физ.-мат. науки, 2019. Т. 23, № 3. С. 541-581. doi: 10.14498 /vsgtu1664.

\section{Сведения об авторах}

Гудрет Исфандияр Келбалиев (1) https://orcid.org/0000-0002-6275-3732

доктор технических наук; член-корреспондент НАН Азербайджана; заведующий отделом; отд. моделирования и технологии химических и экологических процессов;

e-mail: kudret.kelbaliev@mail.ru

Сакит Рауф Расулов (1) https://orcid.org/0000-0002-1548-3143

доктор технических наук, профессор; заведующий кафедрой; каф. промышленной безопасности и охраны труда; e-mail: rasulovsakit@gmail.com 
и интегро-дифференциального кинетического уравнения коалесценции и дробления для скачкообразного изменения размеров частиц. Предложено множество аналитических решений этих уравнений для частных случаев. Более глубокий анализ на основе математических закономерностей явлений переноса позволяет стандартным образом рассчитывать такие системы в некотором приближении как непрерывные с бесконечно малым скачком. Показано, что детерминированное описание этих явлений без учета их стохастической природы является неполным и может приводить к существенным отклонениям от истинной природы указанных выше процессов.

Полученные результаты сравнены с существующими экспериментальными данными по коалесценции и дроблению капель и пузырей, что показало удовлетворительное соответствие расчетным значениям.

Ключевые слова: массоперенос, межфазная пленка, диссипация энергии, изотропная турбулентность, поверхностное натяжение, агрегативная устойчивость.

Получение: 14 декабря 2018 г. / Исправление: 7 марта 2019 г. Принятие: 10 июня 2019 г. / Публикация онлайн: 22 октября 2019 г.

Введение. Физические явления коалесценции и дробления капель и пузырей в турбулентном потоке составляют основу многих процессов химической, нефтеперерабатывающей, пищевой и фармацевтической технологий и связаны в основном с изменением спектра и числа частиц в единице объема, определяющих величину межфазной поверхности массо- и теплопереноса. Коалесценция капель и пузырей, связанная с их столкновением и последующим укрупнением, широко используется в процессах разделения и расслоения фаз (осаждение, всплытие) в эмульсиях, суспензиях и в других многофазных средах.

Дробление капель и пузырей, необходимое для увеличения межфазной поверхности, достаточно широко используется в массообменных процессах жидкостной экстракции, абсорбции, в газожидкостных реакторах, в процессах распыления и горения и т. д. Сущность процессов коалесценции и дробления капель и пузырей состоит в потере агрегативной, а в некоторых случаях и седиментационной устойчивости дисперсной системы в целом, под действием внешних сил или же в самопроизвольных изменениях в системе из-за стремления уменьшить избыточную поверхностную энергию.

Теоретические и экспериментальные исследования процессов коалесценции, дробления и деформации капель и пузырей приведены во многих работах [1-16]. Первые теоретические исследования явлений коалесценции двухчастичных столкновений капель весьма малых размеров в приближении броуновских частиц в однородной неограниченной системе на простейших моделях были проведены Смолуховским [1,2], в результате чего определены частота и время коалесценции, обратно пропорциональные коэффициенту молекулярной диффузии броуновских частиц. Последующие исследования явлений коалесценции и дробления капель и пузырей, протекающих в трубах, в колонных аппаратах и в перемешивающих устройствах, были посвящены экспериментальному $[3-5,7,10]$, теоретическому исследованию и динамике этих явлений в турбулентном потоке [9, 12-19]. В этих работах представлены модели и характерный механизм процессов, полуэмпирические формулы для 
расчета минимальных и максимальных размеров частиц, частоты и времени коалесценции и дробления в зависимости от коэффициента турбулентной диффузии, диссипации энергии и свойств частиц и потока. Основными характеристиками этих процессов являются частота коалесценции (или столкновения) и дробления, определяемая в изотропном турбулентном потоке диссипацией энергии и масштабом турбулентности, а также свойства и размеры самих частиц и среды (плотность, вязкость, поверхностное натяжение), и в большинстве случаев состояние дисперсной системы определяется минимальными и максимальными размерами капель и пузырей $[5-7,12,15,19]$.

В работе [20] приведен литературный обзор процессов дробления капель и пузырей в турбулентном потоке, где основное внимание уделено анализу механизмов дробления и некоторым вопросам определения частоты, а также ее зависимости от удельной диссипации энергии, характеристик среды и размеров частиц. Минимальные размеры частиц характеризуют такое состояние дисперсной системы, которая более склонна к коалесценции, а максимальные размеры характеризуют состояние системы, более склонной к деформации и дроблению капель и пузырей.

Многие работы посвящены процессам коалесценции и дробления в турбулентном потоке [9,21-23], где исследованы влияние параметров изотропной турбулентности на процессы коалесценции и дробления капель и пузырей в системах «газ - жидкость» и «жидкость - жидкость», влияние свойств среды и капель на протекание процессов и на распределение капель [24], влияние концентрации частиц на коалесценцию и дробление в многофазных средах [25] и другие проблемы расчета этих процессов [26-28].

Другой характеристикой дисперсной системы, в которой протекают процессы коалесценции и дробления, является скорость изменения размеров и числа частиц, другими словами, эволюция функции распределения по размерам и по времени, теоретические и экспериментальные исследования которых приведены в работах [29-35].

Важными характеристиками изотропного турбулентного потока являются колмогоровский масштаб турбулентности $\lambda_{0}$ и диссипация энергии на поверхности капли $E_{R}$, определяемые в виде

$$
\lambda_{0}=\left(\frac{\nu_{c}^{3}}{\varepsilon_{R}}\right)^{0.25}, \quad E_{R} \sim \frac{\pi a^{3}}{6} \tau_{P} \sim \frac{\pi a^{2} \eta_{c}}{6} \sqrt{\frac{\rho_{c}\left(\varepsilon_{R} a\right)^{2 / 3}}{\rho_{d}}}
$$

где

$$
\tau_{P}=\frac{\eta_{d} \bar{U}}{a} \sim \frac{\eta_{d}\left(\varepsilon_{R} a\right)^{1 / 3}}{a}
$$

- вязкостное напряжение на поверхности капли;

$$
\bar{U}^{2}(r)=C_{0}\left(\varepsilon_{R} r\right)^{2 / 3}
$$

- среднеквадратичная скорость турбулентного потока.

Следует отметить, что в процессах коалесценции и дробления капель и пузырей в изотропном турбулентном потоке немаловажную роль играет коэффициент турбулентной диффузии, определямый для различных областей значений масштаба турбулентных пульсаций $\lambda$ в виде [1]

$$
\begin{aligned}
& \lambda>\lambda_{0}, \quad D_{T}=\alpha_{0}\left(\varepsilon_{R} \lambda\right)^{1 / 3} \lambda \\
& \lambda<\lambda_{0}, \quad D_{T}=\alpha_{0}\left(\varepsilon_{R} / \nu_{c}\right)^{0.5} \lambda^{2} .
\end{aligned}
$$


Вместе с тем, учитывая степень увлечения частиц пульсирующей средой, для коэффициента турбулентной диффузии частиц можно написать [36]

$$
D_{T P} \approx \mu_{p}^{2} D_{T}
$$

где коэффициент $\mu_{p}^{2}$ - степень увлечения частиц турбулентным потоком, зависящий от размеров частиц, причем с их ростом можно предположить, что $\mu_{p}^{2} \rightarrow 0$.

В более широком смысле в работе [37] на основе имеющихся экспериментальных исследований предложены эмпирические формулы для определения коэффициентов диффузии частиц в зависимости от динамической скорости потока и скорости осаждения и т. д.

Целью данной работы является теоретический анализ существующих принципов исследования процессов коалесценции и дробления капель и пузырей в изотропном турбулентном потоке с присущими им свойствами.

1. Максимальные и минимальные размеры частиц. Дисперсные системы (эмульсии, суспензии) в большей степени характеризуются полидисперсностью размеров частиц, колеблющихся в широких пределах - от 1 мкм до 200 мкм, хотя в потоке могут встретиться частицы коллоидных размеров и более крупные частицы. Однако состояние дисперсного потока, его агрегативная устойчивость к изменению размеров и седиментационная устойчивость к осаждению в целом определяющие структуру спектра дисперсий в объеме, характеризуется минимальными и максимальными размерами частиц. Следует отметить, что процессы, протекающие в дисперсных системах, сопровождаются не только столкновением и укрупнением сталкивающихся капель, но и обратным явлением - дроблением, вызванным тем, что сильно взаимодействовавшие частицы разлетаются на осколки либо не могут сохранять устойчивое состояние и распадаются самопроизвольно или под действием каких-либо возмущений на их внешней поверхности. Таким образом, в дисперсных системах существует некоторый размер $a_{\max }$, выше которого капли неустойчивы, деформируются и мгновенно разрушаются, и минимальный размер $a_{\min }$, определяющий нижний порог устойчивости капель, т. е. при определенных условиях течения капли, достигшие этих размеров, не могут дальше дробиться. Максимальный размер частиц характеризует неустойчивое состояние капель и пузырей, зависящих от гидродинамических условий течения дисперсной среды, и при определенный условиях турбулентного течения проявляется склонность к распаду и дроблению единичной капли. В литературе имеется множество формул для оценки максимальных значений капель в турбулентном потоке.

Так, для газовых пузырей важно отметить следующие выражения в области чисел $\operatorname{Re}_{d}<100$ :

$$
\begin{array}{ll}
a_{\max }=\frac{\sigma^{0.6}}{\rho_{c}^{0.4} \rho_{d}^{0.2}} \varepsilon_{R}^{-0.4} & \text { из работы [1], } \\
a_{\max }=0.725\left(\sigma / \rho_{c}\right)^{0.6} \varepsilon_{R}^{-0.4} & \text { из работы [38], } \\
a_{\max }=\lambda_{f}\left(\frac{\sigma}{\varepsilon_{R}^{2 / 3} \rho_{c} \lambda_{f}^{5 / 3}}\right)^{0.926} & \text { из работы [40]. }
\end{array}
$$


Для жидких капель, если $\eta_{c} \neq \eta_{d}$, и для области чисел $\mathrm{Re}_{d}<10$ предложены следующие выражения для максимальных значений:

$$
\begin{aligned}
a_{\max } & =1.12\left(\frac{\sigma}{\rho_{c}}\right)^{0.6}\left(\frac{\eta_{c}}{\eta_{d}}\right)^{0.1} \varepsilon_{R}^{-0.4} \quad \text { из работы [21], } \\
a_{\max } & =\frac{\sigma \nu_{c}^{0.5}}{\eta_{c} \varepsilon_{R}^{0.5}} \frac{16\left(\eta_{d} / \eta_{c}\right)+16}{19\left(\eta_{d} / \eta_{c}\right)+16} \quad \text { из работы [39], } \\
a_{\max } & =C^{\frac{5}{3+2 \alpha}} L\left(\frac{\sigma}{\rho_{c} \varepsilon_{R}^{2 / 3} L^{5 / 3}}\right)^{\frac{3}{3+2 \alpha}},
\end{aligned}
$$

где $\lambda_{f}$ - начальная длина масштаба турбулентности; $L$ - интегральный масштаб турбулентности; $\alpha$ - коэффициент, причем при $\alpha=1$ последнее выражение совпадает с формулой, предложенной в работе [38]. Вычисление по этим формулам при одинаковых условиях течения дает существенный разброс численных значений максимального размера капель [41].

С целью оценки минимальных и максимальных размеров капель воспользуемся условием равенства динамической силы, действующей на поверхности капель

$$
F_{D}=C_{D} \frac{\rho_{c} U^{2}}{2}
$$

(где $C_{D}=C_{D}\left(\mathrm{Re}_{d}\right)$ - коэффициент сопротивления капель и пузырей [42-44]; $\operatorname{Re}_{d}=U a / \nu_{c}$ - число Рейнольдса для частицы, определяемое через среднюю скорость турбулентного потока $U$ ) с силой поверхностного натяжения $F_{\sigma}=$ $=4 \sigma / a_{s}$ [1]. Здесь предполагается, что динамический напор действует на определенную часть поверхности капли и поэтому может быть связан с коэффициентом сопротивления. Из условия равенства этих сил имеем

$$
C_{D} \frac{\rho_{c} U^{2}}{2}=\frac{4 \sigma}{a_{s}}
$$

С учетом выражения для пульсационной скорости

$$
V^{\prime}=\gamma\left(\frac{\rho_{d}}{\rho_{c}} \varepsilon_{R} \lambda\right)^{1 / 3}
$$

определим выражение для максимального размера капель или пузырей в виде $[12-14]$

$$
a_{\max }=\gamma^{-1.2}\left(\frac{8}{C_{D}}\right)^{0.6} \frac{\sigma^{0.6}}{\rho_{c}^{0.2} \rho_{d}^{0.4}} \varepsilon_{R}^{-0.4} .
$$

Здесь $\gamma$ - коэффициент, определяемый экспериментальным путем. Исходя из уравнения (4) для различных областей изменения числа $\operatorname{Re}_{d}$ можно получить ряд формул для оценки максимального размера капель $\left(C_{D} \approx 8 / 3\right)$, в частности вида

$$
a_{\max } \approx 1.93 \gamma^{-1.2} \frac{\sigma^{0.6}}{\rho_{c}^{0.2} \rho_{d}^{0.4}} \varepsilon_{R}^{-0.4}, \quad 2 \cdot 10^{3} \leqslant \operatorname{Re}_{d} \leqslant 5 \cdot 10^{5}
$$

Для пузырей воздуха в воде в области их деформации $500<\mathrm{Re}_{d} \leqslant 2000$, где коэффициент сопротивления, согласно экспериментальным данным [42], 
составляет величину $C_{D} \approx 14.5 \mathrm{Mo}^{1 / 2} \operatorname{Re}_{d}^{4 / 3}$, на основе выражения (4) для максимальных размеров, получим [14]

$$
a_{\max }=0.82 \gamma^{-2 / 3} \mathrm{Mo}^{-1 / 6}\left[\left(\frac{\nu_{c}}{U}\right)^{-4 / 9}\left(\frac{\sigma}{\rho_{c}^{1 / 3} \rho_{d}^{2 / 3}}\right)\right]^{1 / 3} \varepsilon_{R}^{-2 / 9},
$$

где Мо $=\frac{g \eta_{c}^{4}}{\rho_{c} a^{3}} \frac{\Delta \rho}{\rho_{c}}-$ число Мортона.

Если число Мортона Мо $>10^{-7}$ и $0.1<\operatorname{Re}_{d}<100$, то согласно [1] и экспериментальным данным [10] получим $C_{D}=16 / \operatorname{Re}_{d}$ и для максимального размера пузырей получим соотношение

$$
a_{\max } \approx 0.354 \gamma^{-3}\left(\frac{U}{\nu_{c}}\right)^{1.5} \frac{\sigma^{1.5}}{\rho_{c}^{0.5} \rho_{d}} \varepsilon_{R}^{-1}
$$

Если число Мортона Мо $<10^{-7}$ и $0.1 \leqslant \operatorname{Re}_{d}<100$, то можно использовать уравнение (7) с коэффициентом 0.192, и, если Мо $<10^{-7}$ и $100 \leqslant \operatorname{Re}_{d}<400,-$ с коэффициентом, равным 0.068. В частности, при $2<\operatorname{Re}_{d}<10^{3}$ для пузырей можно положить $C_{D} \approx 14 / \operatorname{Re}_{d}^{0.5}$, в результате чего выражение (3) принимает вид

$$
a_{\max } \approx 0.619 \gamma^{-12 / 7}\left[\left(\frac{U}{\nu_{c}}\right)^{0.5}\left(\frac{\sigma}{\rho_{c}^{1 / 3} \rho_{d}^{2 / 3}}\right)\right]^{6 / 7} \varepsilon_{R}^{-4 / 7} .
$$

Выражение (8) удовлетворительно согласуется с экспериментальными данными [5,6], что показано в работах $[14,15]$.

В литературе также имеется множество формул с использованием критического числа Вебера $\mathrm{We}_{\mathrm{cr}}=a_{\max } \frac{\rho_{c} \bar{U}}{\sigma}$ для определения максимального размера капель и пузырей в турбулентном потоке, среди которых можно отметить следующие:

$$
\begin{array}{ll}
a_{\max }=\left(\frac{\text { We }}{2}\right)^{0.6} \frac{\sigma^{0.6}}{\rho_{c}^{0.4} \rho_{d}^{0.2}} \varepsilon_{R}^{-0.4} & \text { из работы [5], } \\
a_{\max }=\text { We }_{\mathrm{cr}}^{0.6} \frac{\sigma^{0.6}}{\rho_{c}^{0.3} \rho_{d}^{0.3} \eta_{c}^{0.6}} \frac{d_{k}^{1.3}}{U^{1.1}} & \text { из работы }[6], \\
a_{\max } \frac{\rho_{c} U^{2}}{\sigma}\left(\frac{\eta_{c} U}{\sigma}\right)^{0.5}=38\left[1+0.7\left(\frac{\eta_{c} U}{\sigma}\right)^{0.7}\right] & \text { из работы }[31], \\
a_{\max }=\left(\frac{\text { We }}{2}\right)^{0.6} \sigma^{0.6} \rho_{c}^{0.2} \varepsilon_{R}^{-0.4}, & \text { из работ }[45,46] .
\end{array}
$$

Здесь $d_{k}$ - диаметр канала.

В литературе можно встретить и другие эмпирические формулы для определения максимального размера капель:

$$
\begin{aligned}
& a_{\max }=A_{1}\left(\sigma+A_{2} \eta_{c} \varepsilon_{R}^{1 / 3} a^{1 / 3}\right)^{0.6} \rho_{c}^{-0.6} \varepsilon_{R}^{-0.4}, \quad A_{1} \approx 1.9, \quad A_{2} \approx 0.35 \\
& \frac{\rho_{c} \varepsilon^{0.4} a^{5 / 3}}{\sigma}=A_{1}^{5 / 3}\left(1+A_{2}\left(\frac{\rho_{c}}{\rho_{d}}\right)^{0.5} \frac{\eta_{c} \varepsilon_{R}^{1 / 3} a^{1 / 3}}{\sigma}\right),
\end{aligned}
$$


где $A_{1}, A_{2}$ - коэффициенты, имеющие различные значения в зависимости от вязкости среды.

Минимальный размер частиц в изотропном потоке характеризует состояние капли или пузыря, гидродинамически устойчивое к дроблению при определенных условиях течения потока, а при большой концетрации частицсклонность к их интенсивному столкновению и коалесценции, что также определяется условием (4). Учитывая для изотропного турбулентного потока

$$
V_{\lambda}=\gamma_{0}\left(\frac{\rho_{d}}{\rho_{c}} \varepsilon_{R} \lambda\right)^{1 / 3}, \quad \varepsilon_{R}=\nu_{c}^{3} / \lambda_{0}^{4},
$$

а также выражение (4) (но для минимальных размеров капель и пузырей), получим [15]

$$
a_{\min }=\frac{C_{D}}{8} \gamma^{2}\left(\rho_{c} \rho_{d}^{2}\right)^{1 / 3} \frac{\nu_{c}^{2}}{\sigma} .
$$

Следует отметить, что в работе [1] для минимального размера капель получена более простая формула, учитывающая плотность среды и коэффициент сопротивления:

$$
a_{\min } \approx \rho_{c} \nu_{c}^{2} / \sigma
$$

Поскольку коэффициент сопротивления зависит от числа $\mathrm{Re}_{d}$, paccмотрим различные варианты [43]:

$$
\begin{array}{ll}
a_{\min } \approx \sqrt{3} \gamma\left(\frac{\nu_{c}^{3}}{U \sigma}\right)^{0.5}\left(\rho_{c} \rho_{d}^{2}\right)^{1 / 6}, \quad \operatorname{Re}_{d}<0.1 \\
a_{\min } \approx \sqrt{2} \gamma\left(\frac{\nu_{c}^{3}}{U \sigma}\right)^{0.5}\left(\rho_{c} \rho_{d}^{2}\right)^{1 / 6}, \quad 0.1 \leqslant \operatorname{Re}_{d} \leqslant 100
\end{array}
$$

Для области $400 \leqslant \mathrm{Re}_{d} \leqslant 2 \cdot 10^{3}$ согласно экспериментальным данным [38] для капли жидкости в воздухе можно получить следующую корреляцию:

$$
C_{D}=14.5 \mathrm{Mo}^{-1.5} \operatorname{Re}_{d}^{4 / 3}
$$

учитывая которую, можно написать [43]:

$$
a_{\min }=0.168 \gamma^{-2 / 3} \mathrm{Mo}^{-1.5} \frac{\sigma^{3}}{U^{4} \rho_{c} \rho_{d}^{2} \nu_{c}^{2}} \text {. }
$$

Для воздушных пузырьков в воде при $\mathrm{Re}_{d}>2 \cdot 10^{3}$ значение коэффициента сопротивления устанавливается на уровне $C_{D}=8 / 3$, и

$$
a_{\min }=\frac{1}{3} \gamma^{2} \frac{\nu_{c}^{2}}{\sigma}\left(\rho_{c} \rho_{d}^{2}\right)^{1 / 3} \text {. }
$$

В перемешивающих устройствах минимальный размер капель определяется выражением [19]

$$
a_{\min }=0.5\left(n d_{T}\right)^{-1.75}\left(\frac{\sigma^{3} \nu_{c}}{\rho_{c} \rho_{d}^{2}}\right)^{0.25},
$$


где $n$ - угловая частота вращения мешалки, $d_{T}$ - диаметр мешалки. Как следует из этого уравнения, минимальный размер капель определяется характеристиками мешалки: угловой скоростью вращения и размером перемешивающего устройства, а также свойствами среды и капли. В табл. 1 приведено сравнение экспериментальных данных $[19,47]$ с расчетными значениями минимальных капель масла в воде, полученными по формуле (14); значения параметров для расчета: $\sigma=72.2 \cdot 10^{-3} \mathrm{H} / \mathrm{M}, \rho_{c}=1000 \mathrm{K \Gamma} / \mathrm{M}^{3}, \rho_{d}=850 \mathrm{K \Gamma} / \mathrm{m}^{3}$, $\nu_{c}=10^{-6} \mathrm{~m}^{2} /$ сек, $d_{T}=0.027 \mathrm{~m} ; \operatorname{Re}_{d}=n d_{T} a / \nu_{c} ; U=n d_{T}$.

Таблица 1

Сравнение экспериментальных [47] и рассчитанных по формуле (14) значений минимальных размеров капель масла в воде [Comparison of experimental and calculated values of the minimum size of oil droplets in water]

\begin{tabular}{c|c|c|c|c}
\hline \multirow{2}{*}{$\operatorname{Re}_{d}$} & \multirow{2}{*}{$n, \mathrm{sec}^{-1}$} & \multirow{2}{*}{$U, \mathrm{~m} / \mathrm{sec}$} & \multicolumn{2}{|c}{ Minimum droplet size $\left(a_{\min } \times 10^{6}\right), \mathrm{m}$} \\
\cline { 4 - 5 } & & & experimental data $[47]$ & calculated by Eq. (14) \\
\hline 22.50 & 16.67 & 0.450 & 53.0 & 54.2 \\
19.44 & 20.0 & 0.540 & 39.0 & 39.4 \\
16.87 & 25.0 & 0.675 & 26.0 & 26.6 \\
14.58 & 30.0 & 0.810 & 19.0 & 19.4 \\
13.23 & 35.0 & 0.945 & 15.0 & 14.7 \\
11.25 & 41.67 & 1.125 & 10.5 & 10.9 \\
\hline
\end{tabular}

Parameter values for calculation: $\sigma=72.2 \cdot 10^{-3} \mathrm{~N} / \mathrm{m}, \rho_{c}=1000 \mathrm{~kg} / \mathrm{m}^{3}, \rho_{d}=850 \mathrm{~kg} / \mathrm{m}^{3}$, $\nu_{c}=10^{-6} \mathrm{~m}^{2} / \mathrm{sec}, d_{T}=0.027 \mathrm{~m} ; \operatorname{Re}_{d}=n d_{T} a / \nu_{c} ; U=n d_{T}$.

Приведенные корреляции для расчетов минимальных и максимальных размеров деформируемых частиц (капель, пузырей) с определенной точностью могут быть использованы для решения задач коалесценции, дробления и разделения дисперсных систем.

Многочисленные теоретические и экспериментальные исследования состояния деформируемых частиц в турбулентном потоке [48-51] позволили предложить различные формулы для оценки максимальных и минимальных размеров дисперсных включений, причем, как показали исследования, эти размеры зависят прежде всего от удельной диссипации энергии, свойств среды и частиц, от коэффициента сопротивления частиц, а в некоторых случаях и от критического значения числа Вебера We $\mathrm{cr}[6,42]$. Использование коэффициента сопротивления в уравнении равновесия (3) позволяет расширить область адекватного описания максимальных и минимальных размеров частиц. Сравнивая расчетные данные на основе приведенных формул [4851] с различными экспериментальными измерениями, можно отметить, что максимальный размер капель пропорционален удельной диссипации энергии $\varepsilon_{R}^{-0.4}$ только для области развитой турбулентности при $\mathrm{Re}_{d} \geqslant 2 \cdot 10^{3}$. В остальных случаях степень удельной диссипации энергии может существенно меняться.

Определим отношение максимального размера (4) к минимальному размеру (9) капель для развитой турбулентности при $\operatorname{Re}_{d}>2000$ в виде

$$
\frac{a_{\max }}{a_{\min }} \sim C_{D}^{-1.6}\left(\frac{\mathrm{Ke}}{\mathrm{Mo}}\right)^{0.4} \frac{\rho_{c}}{\rho_{d}},
$$

где $\mathrm{Ke}=\frac{g \sigma}{\eta_{c} \varepsilon_{R}}-$ безразмерное число, характеризующее отношение поверх- 
ностной энергии к энергии турбулентного потока [52] и определяющее степень агрегативной устойчивости капель и пузырей. Если число Ke $\gg 1$ или $\varepsilon_{R} \gg g \sigma / \eta_{c}$, то в этом случае капля считается устойчивой и не подвергается дроблению в турбулентном потоке. В работе [15] на основе экспериментальных данных $[2,42]$ предложено условие $\operatorname{Re}_{d} \mathrm{Mo}^{1 / 6}<7$, при котором пузыри не подвергаются значительной деформации, при этом Мo $=\frac{4}{3} C_{D} \mathrm{We}^{3} \mathrm{Re}_{d}^{-4}$.

\section{2. Дробление капель и пузырей в изотропном турбулентном по-} токе. Дробление капель и пузырей в изотропном турбулентном потоке является важным фактором для увеличения межфазной поверхности и скорости тепло- и массопереноса в дисперсных системах. Механизм дробления деформируемых частиц определяется разными факторами. Среди них отметим следующие:

- влияние пульсаций турбулентности определенной частоты на поверхностной части капель и пузырей [8,53-56] на коррекцию формы ${ }^{1}$;

- граничная неустойчивость на поверхности капли, определяемая турбулизацией пограничного слоя, или общая неустойчивость в результате достижения размеров капли максимального значения $a \geqslant a_{\max }$;

- воздействие внешней среды ${ }^{2}$, где дробление капель можно определить равновесием между внешними силами непрерывной фазы и силами поверхностного напряжениея, способствующими сопротивлению разрушению капли (3) [1, 25, 38];

- результат взаимного упругого столкновения при интенсивном перемешивании системы ${ }^{3}$.

${ }^{1}$ В работах $[1,5]$ флуктуационная частота колебаний поверхности капли с использованием уравнения Рэлея определена в виде

$$
\omega(k)=\left(\frac{2 \sigma}{\pi^{2} \rho_{c} a^{3}} \frac{(k+1)(k+2) k(k-1)}{(k+1) \rho_{d} / \rho_{c}+1}\right)^{0.5},
$$

где $k$ - волновое число. При $k=2$ из данной формулы вытекают формулы для определения частоты, соответствующие дроблению пузырей

$$
\omega(a)=\frac{2 \sqrt{6}}{\pi}\left(\frac{\sigma}{\rho_{c} a^{3}}\right)^{0.5}, \quad \rho_{d} \ll \rho_{c}
$$

и капель

$$
\omega(a)=\frac{4}{\pi}\left(\frac{\sigma}{\rho_{d} a^{3}}\right)^{0.5}, \quad \rho_{d} \gg \rho_{c} .
$$

В итоге для небольших деформаций формулу капли можно определить суперпозицией «линейных гармоник»:

$$
r(t, \theta)=R\left[1+\sum_{k} A_{k} \cos \left(\omega_{k} t\right) P_{k}(\cos \theta)\right]
$$

где $P_{k}(\cos \theta)$ - функции Лежандра, $A_{k}$ - коэффициенты ряда, определяемые как $A_{k}=$ $=A_{k 0} \exp \left(-\beta_{k} t\right), \beta_{k}-$ коэффициент затухания

$$
\beta_{k}=\frac{(k+1)(k-1)(2 k+1) \eta_{d}+k(k+2)(2 k+2) \eta_{c}}{\left(\rho_{d}(k+1)+\rho_{c} k\right) R^{2}} .
$$

\footnotetext{
2 Данное условие также характеризует деформацию формы капель и пузырей.

${ }^{3}$ Важно отметить, что не всякое столкновение капель и пузырей приводит к их слиянию и коалесценции; при упругом столкновении капля может распадаться на осколки, тем са-
} 
Общий обзор по дроблению капель и пузырей приведен в работе [20], где рассмотрены вопросы, связанные с частотой дробления и характером функции распределения частиц по размерам, хотя не рассмотрены анализ максимальных и минимальных размеров и характерные особенности влияния вторичных процессов дробления на изменение функции мультимодального распределения капель. Несмотря на множество механизмов дробления капель и пузырей, важным параметром, характеризующим этот процесс, является частота дробления в турбулентном потоке, определению которой посвящено множество работ [3, 11, 13-15, 20, 25,33,47].

В работе [3] на основе анализа поверхностной и кинетической энергии турбулентного потока для частоты дробления капель предложено следующее выражение:

$$
\omega(a)=C_{1} a^{-2 / 3} \varepsilon_{R}^{1 / 3} \exp \left(-\frac{C_{2} \sigma}{\rho_{c} \varepsilon_{R}^{2 / 3} a^{5 / 3}}\right) .
$$

В работах [12-14] путем аналитического решения уравнения массопереноса с учетом выражений для турбулентной диффузии частиц

$$
\frac{\partial N}{\partial t}=\frac{1}{r^{2}} \frac{\partial}{\partial r}\left(r^{2} D_{T P} \frac{\partial N}{\partial r}\right)
$$

$t=0, r>R, N=N_{0}, t>0, r=R, N=0, t>0, r \rightarrow \infty, N=N_{0}$, предложено аналогичное (16) уравнение для частоты дробления для случая $\lambda>\lambda_{0}$ (где $C_{1}=C_{10} N_{0} a^{3}$ ), когда коэффициент турбулентной диффузии определяется по первой формуле (2), причем время дробления капель принимается в виде $\tau \sim \sigma /\left(\rho_{c} \varepsilon_{R} a\right)$, хотя в работах [33,34] он определяется как $\tau \sim a^{2 / 3} \varepsilon_{R}^{-1 / 3}$. Для случая $\lambda<\lambda_{0}$, т. е. для вязкого течения с использованием второго уравнения (2), частоту дробления можно определить следующим выражением:

$$
\omega(a)=C_{01} N_{0} a^{3}\left(\frac{\varepsilon_{R}}{\nu_{c}}\right)^{0.5} \exp \left(-C_{02} \frac{\sigma}{\left(\nu_{c} \varepsilon_{R}\right)^{1 / 2} a \rho_{c}}\right) .
$$

Как следует из этого уравнения, частота дробления капель и пузырей в вязкой области или в жидкой среде обратно пропорциональна вязкости среды $\sim \nu_{c}^{-0.5}$. В ряде работ в зависимости от механизма дробления предложены следующие формулы для частоты дробления:

$$
\begin{array}{ll}
\omega(a)=C_{3} a^{-2 / 3} \varepsilon_{R}^{1 / 3}\left(\frac{2}{\sqrt{\pi}}\right) \Gamma\left(\frac{3}{2} \frac{C_{4} \sigma}{\rho_{c} \varepsilon_{R}^{2 / 3} a^{5 / 3}}\right) & \text { в [20,57,58]; } \\
\omega(a)=\frac{K_{0}}{a}\left(8.2 \varepsilon_{R}^{2 / 3} a^{2 / 3}-\frac{\sigma}{\rho_{c} a}\right)^{0.5} & \text { в [33]; } \\
\omega(a)=C_{5} \varepsilon_{R}^{1 / 3} \operatorname{erfc}\left[\left(C_{6} \frac{\sigma}{\rho_{c} \varepsilon_{R}^{2 / 3} a^{5 / 3}}+C_{7} \frac{\eta_{d}}{\rho_{c}^{0.5} \rho_{d}^{0.5} \varepsilon_{R}^{1 / 3} a^{4 / 3}}\right)^{0.5}\right] & \text { в }[20,59] ; \\
\omega(a)=\frac{a^{5 / 3} \varepsilon_{R}^{19 / 15} \rho_{c}^{1.4} \exp \left(-\frac{\sqrt{2} \sigma^{1.8}}{a^{3} \rho_{c}^{1.8} \varepsilon_{R}^{1.2}}\right)}{\sigma^{1.4}} & \text { в [20,60]; } \\
\omega(a)=C_{8} n \operatorname{erfc}\left[\left(C_{9} \frac{\sigma^{1.5}}{n^{3} d_{T}^{3} \rho_{c}^{1.5} a^{1.5}}\right)^{1 / 3}\right] & \text { в }[61] .
\end{array}
$$

мым меняя спектр распределения размеров, хотя отсутствуют работы, свидетельствующие о числе частиц, образовавшихся в результате такого распада. 
Последнее уравнение определяет частоту дробления капель в перемешивающих устройствах и зависит от параметров перемешивания. Для многофазных систем с объемной долей капель $\varphi$ частоту их дробления можно определить в виде $[3,34]$

$$
\omega(a)=C_{10} \frac{\varepsilon_{R}^{1 / 3}}{a^{2 / 3}(1+\varphi)} \exp \left(-C_{11} \frac{(1+\varphi)^{2} \sigma}{\rho_{d} a^{5 / 3} \varepsilon_{R}^{2 / 3}}\right)
$$

Скорость дробления капель в изотропном турбулентном потоке характеризуется константой скорости, определяемой в виде [50]

$$
\begin{aligned}
& \operatorname{Re}_{d}<1, \quad k_{R}=A_{0} \frac{\varepsilon_{R}^{1 / 3}}{a^{2 / 3}} \exp \left(-\frac{A_{1} \sigma}{\rho_{c} \varepsilon_{R}^{2 / 3} a^{5 / 3}}\right) \\
& \operatorname{Re}_{d}>1, \quad k_{R}=A_{0} \frac{\rho_{c} a^{2 / 3} \varepsilon_{R}^{1 / 3}}{\eta_{c}} \exp \left(-\frac{A_{1} \sigma}{\rho_{c} \varepsilon_{R}^{2 / 3} a^{5 / 3}}\right) .
\end{aligned}
$$

В принципе, выражение, приведенное в скобках, является отношением поверхностной энергии $\left(E_{\sigma} \sim \pi a^{2} \sigma / a \sim \pi a \sigma\right)$ к энергии турбулентного потока $\left(\bar{E}_{T} \sim \pi a^{2}\left(\Delta P_{T}\right), \Delta P_{T}=C_{1} \rho_{c}\left(\varepsilon_{R} a\right)^{2 / 3}\right)$ и характеризует эффективность процесса дробления:

$$
\frac{E_{\sigma}}{\bar{E}_{T}} \sim \frac{\sigma}{\rho_{c} \varepsilon_{R}^{2 / 3} a^{5 / 3}}
$$

Анализируя уравнения (17)-(20), можно отметить, что частота дробления в изотропном турбулентном потоке для области $\lambda>\lambda_{0}$ определяется в основном параметрами турбулентности (удельная диссипация энергии, масштаб турбулентных пульсаций), плотностью среды, поверхностным напряжением, а для вязкого течения $\left(\lambda<\lambda_{0}\right)$ - дополнительно и вязкостью среды.

Важно отметить, что дроблению капель и пузырей в изотропном турбулентном потоке предшествует деформация их формы, причем при значительных числах We и достаточно малых числах Мо они могут принимать формы, не поддающиеся описанию. Условие равновесия между поверхностными силами и внешними силами турбулентного потока (3) может также характеризовать начальные условия деформации частиц. Экспериментальные исследования деформации пузырей при различных числах $\mathrm{We}, \mathrm{Mo}$ и $\mathrm{Re}_{d}$ приведены в работах [10,62], а теоретические исследования представлены работами $[42,44,52,63]$.

3. Коалесценция капель и пузырей в изотропном турбулентном потоке. Коалесценция капель и пузырьков играет важную роль в протекании различных технологических процессов химической технологии и, прежде всего, в уменьшении межфазной поверхности, в расслоении и разделении частиц разных размеров, сопровождающимися их осаждением или всплытием.

Механизм коалесценция капель и пузырей определяется следующими этапами:

- взаимное столкновение частиц с определенной частотой в турбулентном потоке;

- образование межфазной пленки между двумя каплями и ее утончение; 
- разрыв межфазной пленки и дренаж жидкости из одной капли в другую, слияние и образование новой капли.

Взаимные столкновения частиц в объеме потока происходит по различным причинам:

- за счет конвективной броуновской диффузии, мелкодисперсной составляющей частиц, характерной в основном для ламинарного течения при малых числах Рейнольдса;

- за счет турбулентного течения и турбулентной диффузии;

- за счет дополнительных внешних полей (гравитационного, электрического, электромагнитного и т. д. $)^{4}$;

- за счет эффекта зацепления в результате конвективного переноса мелких частиц в окрестности падающей крупной частицы ${ }^{5}$;

- за счет неоднородности полей температуры и давления, способствующих появлению сил, пропорциональных градиентам температуры и давления и действующих в направлении уменьшения этих параметров 6 ;

- кроме указанных явлений, коалесценции способствуют физические явления (испарение капель, конденсация), сопровождающиеся возникновением гидродинамической силы отталкивания (эффект Фасси) испаряющихся капель за счет испарения (стефановский поток) или возникновением силы при конденсационном росте капли, действующей в обратном направлении ${ }^{7}$.

${ }^{4}$ Если колмогоровский масштаб турбулентности $\lambda_{0}$ меньше или сравним с размерами капель в области вязкостного течения, то процесс сопровождается турбулентным блужданием, аналогичным броуновскому, результатом чего является возникновение турбулентной диффузии. Однако турбулентная диффузия может быть характерна для частиц больших размеров на расстояниях, больших $\lambda$, благодаря высокой интенсивности турбулентных пульсаций и неоднородности гидродинамического поля.

${ }^{5}$ В результате осаждения или всплытия крупных частиц за счет образования гидродинамического следа существенно увеличивается захват мелких частиц крупными, что приводит к гравитационной коалесценции, если они падают вдоль линии, близкой к линии центров. В процессе коалесценции капель немаловажную роль играет коэффициент захвата, определяющий отклонение реального сечения захвата частиц от геометрического [32]

$$
\vartheta=\frac{I}{\pi(L+R)^{2} N_{0} V_{\infty}}
$$

где $I$ - массовый поток к поверхности выделенной частицы, $\vartheta-$ коэффициент захвата, $L-$ характерный масштаб расстояния, $V_{\infty}$ - скорость невозмущенного сферой течения среды. Связь между числом Шервуда и коэффициентом захвата при конвективной диффузии имеет вид

$$
\mathrm{Sh}=\frac{1}{2} \operatorname{Pe} \vartheta\left(1+\frac{R}{L}\right)^{2} .
$$

Коэффициент захвата определится как [32]

$$
\begin{array}{ll}
\vartheta \approx \frac{4}{\mathrm{Pe}}\left(1-\frac{N_{L}}{N_{0}}\right) b_{*}\left(1+b_{0} \mathrm{Pe}\right), & \mathrm{Pe} \ll 1 ; \\
\vartheta \approx\left(1-\frac{N_{L}}{N_{0}}\right) \mathrm{Pe}^{-2 / 3}\left(1+0.738 \mathrm{Pe}^{-1 / 3}\right), & \mathrm{Pe} \gg 1 .
\end{array}
$$

Здесь $N_{L}$ - концентрация частиц на поверхности сферы радиуса $r=L, b_{*}, b_{0}$ - коэффициенты.

${ }^{6}$ Для мелкодисперсной составляющей дисперсного потока в результате действия этих сил характерна их миграция за счет термодиффузии и бародиффузии, что также способствует их столкновению и коалесценции.

${ }^{7}$ Несмотря на различные механизмы, в процессах химической технологии коалесценция 
Коалесценция капель и пузырей характеризуется следующими этапами:

- сближение и столкновение капель разного размера в турбулентном потоке с образованием межфазной пленки между ними

- утончение и разрыв межфазной пленки под действием внешних сил и деформирующего (расклинивающего) напряжения с последующим дренажом жидкости из одной капли в другую, коалесценцией и укрупнением капель ${ }^{9}$.

При столкновении двух капель образуется межфазная пленка, которая под действием различного рода сил (гравитационных, динамических, капиллярных, молекулярных и т. д.) утончается до некоторой критической толщины и разрывается с дальнейшим слиянием двух капель (рис. 1). В принципе, образовавшаяся между двумя каплями межфазная поверхность обладает конечной кривизной, зависящей от радиусов капель и переменного поверхностного натяжения. Полагая, что в плоской пленке незначительной кривизны кругового сечения течение ламинарное, уравнение переноса импульса в цилиндрических координатах можно записать в виде [52]

$$
-\frac{\partial P}{\partial r}+\frac{\eta}{g r^{2}} \frac{\partial^{2} V_{r}}{\partial \theta^{2}}+\frac{\eta}{g} \frac{\partial^{2} V_{r}}{\partial x^{2}}=0
$$

капель и пузырей в турбулентном потоке составляет основу расчета явлений, протекающих в дисперсных системах «газ - жидкость» (колонны, газо-жидкостные реакторы) и системах «жидкость - жидкость» (перемешивающие устройства) $[4,7,9,12,13,16,17,22,25,29,52]$.

${ }^{8}$ Перенос капель в полидисперсной среде определяется в основном интенсивностью турбулентности потока и гидродинамическими условиями. В условиях изотропной турбулентности частота столкновений капель зависит от удельной диссипации энергии турбулентного потока и свойств среды и дисперсной фазы $[3,13,29,52,54]$. В результате столкновения и фиксирования двух капель с размерами $a_{1}$ и $a_{2}$ между ними образуется межфазная пленка круглой формы (рис. 1), радиус которой определяется в виде [2]

$$
R_{K}=\left(\frac{3 \pi}{4} P_{m}\left(k_{1}+k_{2}\right) a_{r}\right)^{1 / 3}
$$

где $k_{1}, k_{2}$ - коэффициенты упругости капель; $a_{r}=a_{1} a_{2} /\left(a_{1}+a_{2}\right)$ - средний размер капель; $a_{1}, a_{2}$ - диаметры капель; $P_{m}$ - максимальное сжимающее давление. В работе [3] выражения для гидродинамического давления сжатия в турбулентном потоке определены как $P_{m}=\pi a_{r}^{2} \rho_{c} \bar{U}^{2}$, а для изотропной турбулентности $-P_{m} \sim \rho_{c} \varepsilon_{R}^{2 / 3}\left(a_{1} a_{2}\right)^{2} /\left(a_{1}+a_{2}\right)^{4 / 3}$.

${ }^{9}$ Разрыв межфазной пленки способствует слиянию более мелких капель в более крупные, что способствует уменьшению общего числа капель в объеме и нарушению седиментационной устойчивости и характера их распределения.

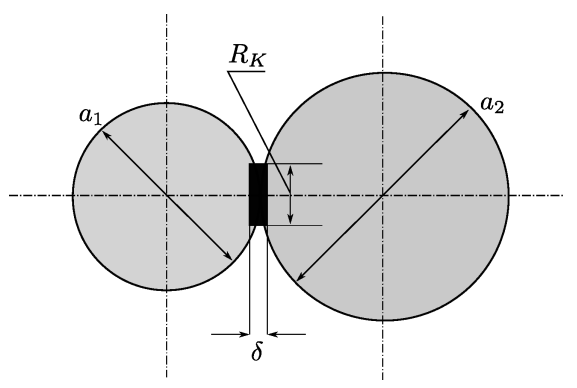

Рис. 1. Столкновение двух капель с образованием межфазной пленки [Figure 1. Collision of two drops with the formation of an interfacial film] 


$$
\begin{aligned}
-\frac{1}{r} \frac{\partial P}{\partial \theta}+\frac{2 \eta}{g r^{2}} \frac{\partial V_{r}}{\partial \theta} & =0 \\
\frac{\partial V_{x}}{\partial x}+\frac{1}{r} \frac{\partial\left(r V_{r}\right)}{\partial r} & =0
\end{aligned}
$$

где $P$ - давление в пленке; $V_{r}, V_{x}$ - составляющие скорости течения в пленке; $\theta$ - полярный угол. Одним из важных краевых условий для решения этих уравнений является условие

$$
x=\delta, \quad-\eta \frac{\partial V_{r}}{\partial r}=\frac{d \sigma}{d r}+\frac{1}{R_{K} \sin \theta} \frac{\partial \sigma(\cos \theta)}{\partial \theta},
$$

определяющее присутствие конвективного течения в жидкой пленке по эффекту Марангони [64,65]. Отметим, что данная проблема с учетом наличия поверхностно-активных веществ, инверсии фаз, эффекта Марангони для одномерного распределения давления в пленке численно решена в работе [65]. Эффект Марангони рассматривается в качестве термокапиллярного течения как результат изменения температуры в пленке и конвективного течения при изменении концентрации или поверхностного натяжения. Преобразуя систему уравнений (21), получим уравнение для изменения толщины межфазной пленки в виде [52]

$$
\frac{d \delta}{d t}=\frac{2 g \Delta P}{3 \pi \eta R_{K}^{4}} \delta^{3}+\frac{2 \delta^{2}}{3 \eta R_{K}^{2} \sin \theta} \frac{\partial \sigma}{\partial \theta} .
$$

Для $\Delta P$ определим следующее выражение:

$$
\Delta P=\left(P_{D}+P_{K}\right) \pi R_{K}^{2}+\Pi,
$$

где $P_{D}, P_{K}$ - динамическое и капиллярное давление $\left(P_{K}=2 \sigma /(g \delta)\right)$, действующие в пленке; П-расклинивающее давление, определяемое как $\Pi=$ $=-A R_{k}^{2} /\left(6 \delta^{2}\right)$ для сферической и $\Pi=-A R_{k}^{2} /\left(6 \delta^{3}\right)$ для деформируемой капель; $A$-выражает константу Ван-дер-Ваальса-Гаммакера $\left(A \sim 10^{-21} J\right)$ $[66,67]$. С учетом вышеизложенного уравнение утончения межфазной пленки (16) представим в виде

$$
\frac{d \delta}{d t}=b_{1} \delta^{3}+b_{2} \delta^{2}-b_{3} \delta, \quad t=0, \quad \delta=\delta_{0},
$$

где

$$
b_{1}=\frac{2 g P_{D}}{3 \eta R_{K}^{4}}, \quad b_{2}=\frac{2}{3 \eta R_{K}^{2}}\left(2 \sigma+\frac{1}{\sin \theta} \frac{\partial \sigma}{\partial \theta}\right), \quad b_{3}=\frac{1}{9} \frac{A a_{r} g}{\pi \eta R_{K}^{4}} .
$$

В работах $[52,68]$ предложены различные аналитические решения данного уравнения:

- для тонких пленок можно положить, что $P_{D} \ll P_{K}-\Pi /\left(\pi R_{K}^{2}\right)$;

- для толстых пленок можно положить $\left(P_{D}+P_{K}\right) \gg \Pi /\left(\pi R_{K}^{2}\right)$.

Для тонких пленок решение уравнения (22) представится как

$$
\delta(t)=\frac{\delta_{0} \exp \left(b_{3} t\right)}{1+\beta_{1} \delta_{0}\left(\exp \left(b_{3} t\right)-1\right)},
$$


где

$$
\beta_{1}=\frac{b_{2}}{b_{3}}=\frac{6 \pi R_{k}^{2}}{A a_{r} g}\left(2 \sigma+\frac{1}{\sin \theta} \frac{\partial \sigma}{\partial \theta}\right) .
$$

Для весьма тонких пленок решение (22) представится как

$$
\delta(t) \approx \delta_{0} \exp \left(-\beta_{3} t\right)
$$

где

$$
\beta_{3}=\frac{2}{3} \frac{P_{D} g \delta_{0}}{\eta_{c} R_{K}^{2}} .
$$

В случае деформируемых капель имеем

$$
\delta(t) \approx \delta_{0}-\beta_{2} t, \quad \beta_{2}=\frac{A g}{9 \pi \eta \delta_{0} R_{K}^{2}} .
$$

Для толстых пленок основными силами, разрывающими толстую межфазную пленку, являются гидродинамические силы, т. е. те силы, которые обусловлены пульсациями скорости. Решение (22) в этом случае можно представить в виде

$$
\begin{array}{ll}
\delta(t)=\frac{\delta_{0}}{\sqrt{1+\beta_{3} t}}, & P_{D} \gg P_{K}, \\
\delta(t)=\frac{\delta_{0}}{1+b_{2} \delta_{0} t}, & P_{D} \ll P_{K} .
\end{array}
$$

Приведенные частные решения (23)-(27) могут быть использованы в инженерных расчетах, в частности при определении толщины межфазной пленки. Исходя из уравнения (23) можно констатировать, что эффект Марангони считается поправкой к коэффициенту поверхностного натяжения в $b_{2}$, хотя он способен оказать значительное влияние на течение и на распределение скорости в указанной пленке. Наличие двумерных давлений, сложность распределения давления на поверхности жидкой пленки и анализ уравнений (21) показывают, что при утончении пленки наличие эффекта Марангони в некоторой степени способствует стабилизации процессов дробления в системе «жидкость - жидкость», т. е. оказывает тормозящий эффект при разрыве пленки.

Как видно из уравнения (23) и формулы определения коэффициента $\beta_{1}$, эффект Марангони приводит к временной стабилизации межфазной пленки, так как в произвольной точке, где за счет действия внешних сил межфазная пленка утончается, происходит локальное усиление поверхностного натяжения, что противодействует утончению. Процесс утончения и, следовательно, разрыва пленки носит случайный характер и, как указано в работе [69], возможность разрыва пленки обратно пропорциональна ее толщине.

В процессе разрушения межфазной пленки существенная роль принадлежит гидродинамическим силам $P_{D}$, порождающим турбулентность, и, прежде всего, высокочастотным турбулентным пульсациям, способствующим ослаблению пленки и межмолекулярных связей между ее основными компонентами, уменьшению прочности и разрыву пленки в результате их растяжения и сжатия, улучшению условий столкновения за счет увеличения частоты столкновений и коалесценции. При одномерном распределении давления 
в пленке с участием поверхностно-активных веществ данная проблема решается также в работе [70].

Экспериментальные данные по утончению межфазной пленки приведены в работах $[65,70]$. На рис. 2 приведены экспериментальные и результаты расчетов по уравнению (23) утончения толщины пленки, причем после достиже-

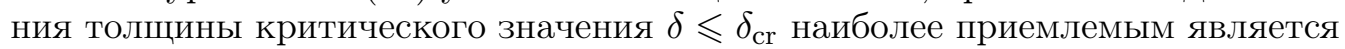
расчет по формуле (24).

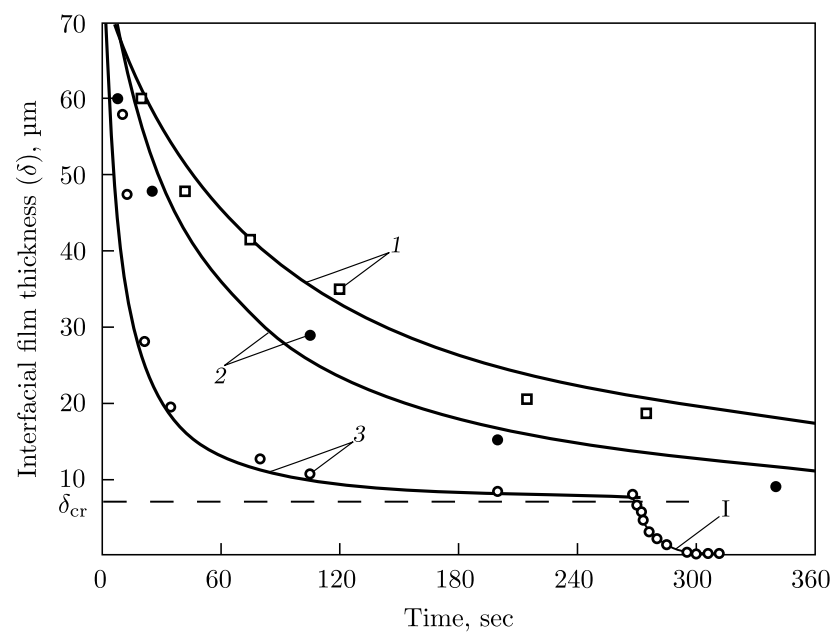

Рис. 2. Сравнение расчетных и экспериментальных данных [70] зависимости толщины межфазной пленки от времени при следующих концентрациях $N_{\mathrm{D}}$ деэмульгатора: $1-N_{\mathrm{D}}=0.2 \mathrm{г} /$ л; $2-N_{\mathrm{D}}=0.5$ г $/$ л; $3-N_{\mathrm{D}}=0.5$ г/л; линии $1-3-$ расчет по уравнению (23); линия I - расчет по уравнению $(24)$

[Figure 2. Comparison of calculated and experimental data [70] of the dependence of the interfacial film thickness on time at the following concentrations $\left(N_{\mathrm{D}}\right)$ of demulsifier: curve 1 corresponds to $N_{\mathrm{D}}=0.2 \mathrm{~g} / \mathrm{l}$; curve 2 corresponds to $N_{\mathrm{D}}=0.5 \mathrm{~g} / \mathrm{l}$; curve 3 corresponds to $N_{\mathrm{D}}=0.5 \mathrm{~g} / \mathrm{l}$; curves $1-3$ correspond to the calculation by Eq. (23); curve I corresponds to the calculation by Eq. (24)]

После разрыва межфазной пленки происходит дренаж жидкости из одной капли в другую, скорость которой определена в работах [52,71]. Рассматривая процесс коалесценции капель в статистическом смысле как обратный процессу их дробления, можно отметить, что частоты коалесценции аналогичны уравнениям (17)-(19) [4, 7, 9, 12, 13, 16, 22, 29, 52].

4. Эволюция функции распределения капель и пузырей в изотропном турбулентном потоке. Эволюция функции распределения капель и пузырей в ограниченном объеме потока, изменяющихся в результате коалесценции и дробления, является важным показателем дисперсной системы и дает ее качественную и количественную характеристику. Основу описания эволюции функции распределения составляют два стохастических уравнения:

- интегро-дифференциальное уравнение коагуляции и дробления, построенное на основе популяционного баланса $[14,32,34,56,59,72]$;

- стохастическое дифференциальное уравнение Фоккера-Планка $[12,13$, $15,32,52,73,74]$. 


\section{1. Интегро-дифференциальное уравнение коагулячии и дроб-} ления. Данное уравнение, описывающее явления коалесценции и дробления капель и пузырей на основе популяционного баланса, является нелинейным интегро-дифференциальным уравнением, имеющим сдвиг по аргументу и зависящим от частоты столкновений и дробления, от переменных размеров (объемов) частиц, от времени эволюции, и может быть представлено в виде

$$
\frac{\partial P\left(v, v^{\prime}\right)}{\partial t}+U_{c} \nabla P\left(v, v^{\prime}\right)=C^{+}+C^{-}+B^{+}+B^{-},
$$

где

$$
C^{+}=\frac{1}{2} \int_{0}^{v} \bar{\beta}\left(v^{\prime}, v-v^{\prime}\right) P\left(v^{\prime}\right) P\left(v-v^{\prime}\right) d v^{\prime}
$$

- выражение, учитывающее рождение новых частиц за определенное время;

$$
C^{-}=-P(v) \int_{0}^{\infty} \bar{\beta}\left(v^{\prime}, v\right) P\left(v^{\prime}\right) d v^{\prime}
$$

- выражение, описывающее уход частиц из данного диапазона размеров;

$$
B^{+}=\int_{0}^{\infty} \varpi\left(v, v^{\prime}\right) P\left(v^{\prime}\right) d v^{\prime}, \quad B^{-}=-\frac{P(v)}{v} \int_{0}^{v} \varpi\left(v^{\prime}, v\right) v^{\prime} d v^{\prime}
$$

- две интегральные составляющие, описывающие приход и уход частиц из данного диапазона в процессе их дробления $[32,74] ; \bar{\beta}\left(v^{\prime}, v\right)$ - частота двухчастичного столкновения частиц объемом $v_{1}$ и $v_{2}$ при их единичной концентрации за промежуток времени $\Delta t ; \varpi\left(v, v^{\prime}\right)$ - функция, характеризующая плотность распределения частиц объема $v$, образовавшихся в единицу времени в результате разрушения частицы объемом $v^{\prime}$. Функция $\bar{\beta}\left(v^{\prime}, v\right)$ является симметричной относительно аргументов, т. е. $\bar{\beta}=\bar{\beta}\left(v_{1}, v_{2}\right), v_{1} \geqslant v_{2}$ и $\bar{\beta}=\bar{\beta}\left(v_{2}, v_{1}\right), v_{2} \geqslant v_{1}$. В отличие от $\bar{\beta}\left(v^{\prime}, v\right)$, функция $\varpi\left(v, v^{\prime}\right)$ является несимметричной функцией, причем исходя из физических соображений можно положить, что $\varpi\left(v, v^{\prime}\right) \geqslant 0$ при $v<v^{\prime}$ и $\varpi\left(v, v^{\prime}\right)=0$ при $v>v^{\prime}$ [32]. В частности, частота столкновения капель в вязкой жидкости определяется симметричной функцией вида [75]

$$
\bar{\beta} \approx 1.294 N_{0}\left(a_{i}+a_{j}\right)^{3}\left(\frac{\varepsilon_{R}}{\nu_{c}}\right)^{0.5},
$$

аналогичной уравнению (17).

В работе [3] для расчета $\bar{\beta}\left(v, v^{\prime}\right)$ используется симметричная функция, аналогичная нормальному распределению:

$$
\bar{\beta}\left(v, v^{\prime}\right)=\frac{2.4}{v^{\prime}} \exp \left(-4.5 \frac{\left(2 v-v^{\prime}\right)^{2}}{v^{\prime 2}}\right) .
$$

Более сложная формула для расчета частоты столкновения частиц (пузырей) с размерами $a_{i}$ и $a_{j}$ предложена в работах $[3,4,77]$ :

$$
\bar{\beta}\left(a_{i}, a_{j}\right)=C_{i j} \varepsilon_{R}^{1 / 3}\left(a_{i}+a_{j}\right)^{2}\left(a_{i}^{2 / 3}+a_{j}^{2 / 3}\right)^{1.5} \lambda\left(a_{i}, a_{j}\right) .
$$


Здесь $C_{i j} \approx 0.28 \div 1.11 ; \lambda\left(a_{i}, a_{j}\right)$ - эффективность коалесценции, которая определяется как [3]

$$
\lambda\left(a_{i}, a_{j}\right)=\exp \left[-C_{11} \frac{\rho_{c} \eta_{c} \varepsilon_{R}}{\sigma^{2}}\left(\frac{a_{i} a_{j}}{a_{i}+a_{j}}\right)^{4}\right]
$$

и уточняется в работе [2] в виде

$$
\lambda\left(a_{i}, a_{j}\right)=\exp \left[-2.3\left(\frac{\rho_{c} \varepsilon_{R}^{2 / 3} a_{i j}^{5 / 3}}{\sigma}\right)^{0.5}\right], \quad a_{i j}=2\left(\frac{1}{a_{i}}+\frac{1}{a_{j}}\right)^{-1} .
$$

В работе [34] для вторичного дробления, т. е. для «дочерних» капель, предлагается следующая формула:

$$
\varpi\left(v, v^{\prime}\right)=\frac{\left(A v^{2 / 9}-B\right)\left(A\left(v^{\prime}-v\right)^{2 / 9}-B\right)}{\int_{v_{\min }}^{v^{\prime}-v_{\min }}\left(A v^{2 / 9}-B\right)\left(A\left(v^{\prime}-v\right)^{2 / 9}-B\right) d v},
$$

где

$$
A=4.1\left(\frac{6}{\pi}\right)^{2 / 9} \rho_{c} \varepsilon_{R}^{2 / 3}, \quad B=\frac{6 \sigma}{(6 / \pi)^{1 / 3} v^{1 / 3}}
$$

Обзор по статистическому анализу процессов дробления капель и пузырей приведен в работах $[20,33,34]$, где также проанализированы разные механизмы дробления капель и пузырей и различные выражения для функций $\bar{\beta}\left(v_{1}, v_{2}\right)$ и $\varpi\left(v, v^{\prime}\right)$. На рис. 3 приведены характерные кривые изменения частоты дробления газовых пузырей в зависимости от размеров, взятые из работ $[33,34,60]$.

Решение уравнения (28) ввиду его нелинейности как в теоретическом, так и в практическом плане представляет определенные трудности. Некоторые частные аналитические решения уравнения дробления

$$
\begin{gathered}
\frac{\partial P\left(v, v^{\prime}\right)}{\partial t}=\int_{0}^{\infty} \varpi\left(v, v^{\prime}\right) P\left(v^{\prime}\right) d v^{\prime}-\frac{P(v)}{v} \int_{0}^{v} \varpi\left(v^{\prime}, v\right) v^{\prime} d v^{\prime}, \\
t=0, \quad P(v, 0)=P_{0}(v) ; \quad v \rightarrow \infty, \quad P(v, t) \rightarrow 0 ; \quad v \rightarrow 0, \quad P(v, t) \rightarrow 0,
\end{gathered}
$$

(учитываются только последние два члена уравнения (28), $C^{+}=C^{-}=0$ ) при постоянном значении $\varpi\left(v, v^{\prime}\right)=$ const и заданном начальном распределении в виде гамма-распределения, а также с использованием метода моментов приведены в работе [32], методом разделения переменных - в работе [14].

В частности, решение этого уравнения можно искать методом разделения переменных в виде $P(v, t)=\psi(t) \varphi(v)$, подставляя которое в $(29)$, получим два уравнения:

$$
\begin{gathered}
\frac{\partial \psi}{\partial t}=-\mu^{2} \psi, \quad \psi(t)=B_{1} \exp \left(-\mu^{2} t\right) \\
\int_{0}^{\infty} \varpi\left(v, v^{\prime}\right) \varphi(v) d v-\frac{\varphi}{v} \int_{0}^{v} v^{\prime} \varpi\left(v, v^{\prime}\right) d v^{\prime}=-\mu^{2} \varphi
\end{gathered}
$$



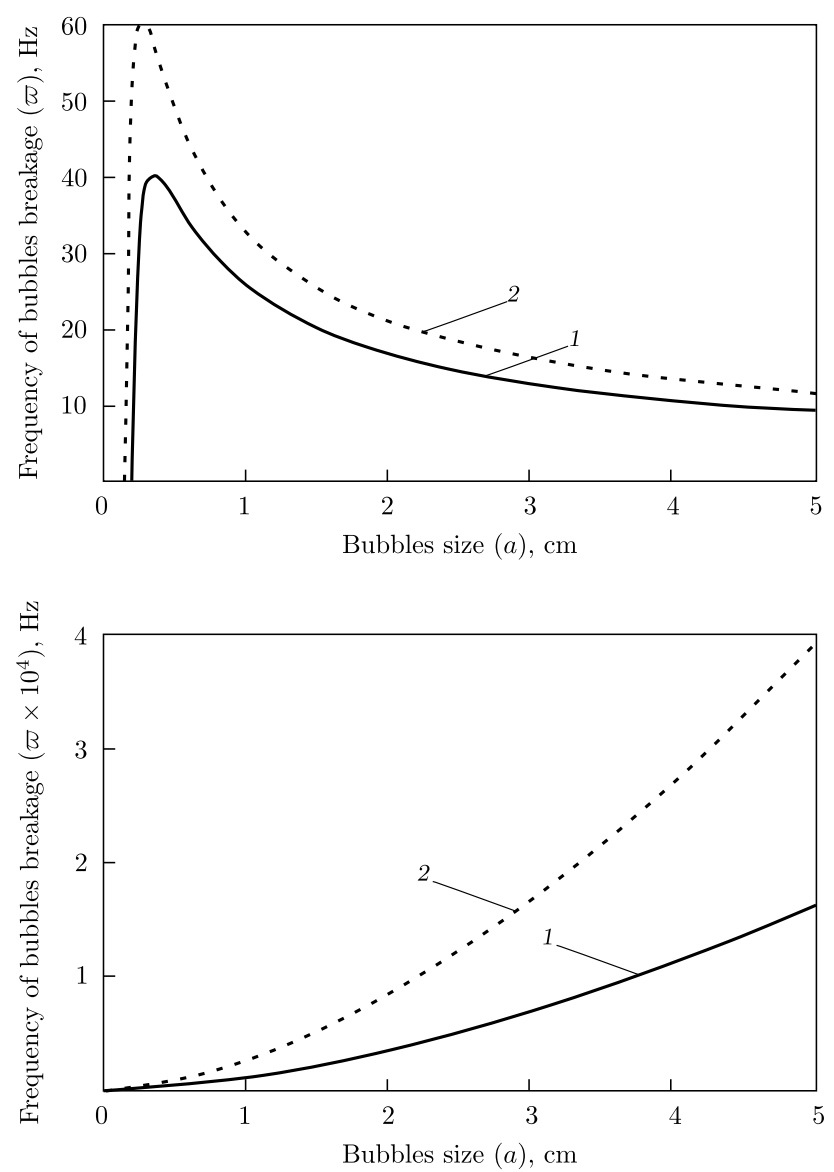

Рис. 3. Зависимость частоты дробления пузырей от их размеров для различных значений диссипации энергии на основе данных работ [33,34] (сверху) и [60] (снизу):

$$
1-\varepsilon_{R}=5.0 \mathrm{~m}^{2} / \mathrm{c}^{3} ; 2-\varepsilon_{R}=10.0 \mathrm{~m}^{2} / \mathrm{c}^{3}
$$

[Figure 3. The dependence of the frequency of bubbles breakage on their sizes for various values of energy dissipation based on the data of Ref. [33,34] (top) and [60] (bottom): curves 1 correspond to $\varepsilon_{R}=5.0 \mathrm{~m}^{2} / \mathrm{sec}^{3}$; curves 2 correspond to $\varepsilon_{R}=10.0 \mathrm{~m}^{2} / \mathrm{sec}^{3}$ ]

Используя краевые условия, общее решение (29) можно представить в виде [14]

$$
P(v, t)=\sum_{n=0}^{\infty} B_{n} \exp \left(-\mu_{n}^{2} t\right) \exp \left(\int \frac{\varpi\left(v, v^{\prime}\right) v^{2}+\alpha(v)-(\partial \alpha / \partial v) v}{\alpha(v) v-\mu_{n}^{2} v^{2}} d v\right)
$$

где $\alpha=\int_{0}^{v} v^{\prime} \varpi\left(v, v^{\prime}\right) d v^{\prime}$. Решение (30) имеет достаточно сложный вид, но при определенном выборе вида функции частоты дробления и начального распределения $P_{0}(v)$ оно существенно упрощается. В частности, если функция частоты дробления задана в виде $\varpi(v)=K_{0} v^{-m}$, то функция распределения 
будет иметь вид

$$
P(v, t)=\sum_{n=0}^{\infty} B_{n} \exp \left(-\mu_{n}^{2} K_{0} t+\int \frac{d v}{v-\mu_{n}^{2}(2-m) v^{m}}\right) .
$$

Если в качестве начального распределения взять функцию $P_{0}(v)=2 \frac{N_{0}}{v_{0}} v e^{-2 v}$, то при $m=1$ будем иметь

$$
P(v, t)=2 \frac{N_{0}}{v_{0}} \exp \left(-2 \frac{v}{v_{0}}-2 \mu_{0}^{2} K_{0} t\right) .
$$

Учитывая условие нормировки $\int_{0}^{\infty} P(v, t) d v=1$ и $v \sim a^{3}$, кумулятивную функцию распределения получим в виде

$$
P_{K}(a, t)=\int_{0}^{a} \frac{P(v, t)}{N_{0}} d a=1-\exp \left(-k_{0} a^{3}\right) .
$$

Это решение удовлетворительно совпадает с экспериментальными данными $[33,34]$ для кумулятивной функции распределения, если $k_{0}=0.00105(x / a)^{2}$ (где $x$ - длина перемещения капли при распылении жидкости под давлением) (рис. 4).

Определенную сложность представляет процесс нахождения решения уравнения коалесценции, полученного из (28) и имеющего сдвиг по аргументу:

$$
\begin{aligned}
\frac{\partial P\left(v, v^{\prime}\right)}{\partial t}=\frac{1}{2} \int_{0}^{v} \bar{\beta}\left(v^{\prime}, v-v^{\prime}\right) P\left(v^{\prime}\right) P\left(v-v^{\prime}\right) d v^{\prime}- & \\
& -P(v) \int_{0}^{\infty} \bar{\beta}\left(v^{\prime}, v\right) P\left(v^{\prime}\right) d v^{\prime} .
\end{aligned}
$$

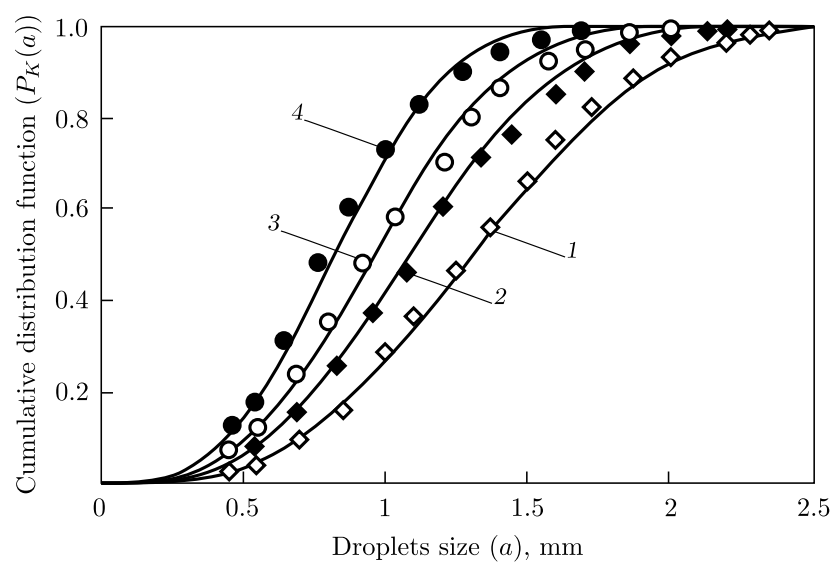

Рис. 4. Изменение кумулятивной функции распределения капель при распылении жидкости на различных расстояниях $x / a: 1-x / a=17.2,2-x / a=22.65,3-$ $x / a=27.39,2-x / a=34.07$

[Figure 4. Change in the cumulative function of the distribution of droplets from spraying liquids at different distances $(x / a)$ : curve 1 corresponds to $x / a=17.2$, curve 2 corresponds to $x / a=22.65$, curve 3 corresponds to $x / a=27.39$, curve 4 corresponds to $x / a=34.07]$ 
Решения (31) сильно зависят от задания начального распределения и функции ядра коалесценции $\bar{\beta}(v)$. Ряд частных аналитических решений $(31)$ для определенных начальных распределений и функции $\bar{\beta}(v)$ приведены в табл. 2.

Численные решения уравнений коалесценции и дробления (29) и (31) приведены в работах $[26,29,32,58,79,80]$, а общий анализ результатов численного и аналитического решений - в работе [32].

4.2. Стохастическое уравнение Фоккера-Планка. Уравнение описывает дисперсные системы с изменением свойств среды и размеров компонентов дисперсной фазы. Так как процессы коалесценции и дробления характеризуются скачкообразностью изменения свойств частиц, для существенного отрезка времени изменение свойств можно считать квазинепрерывным с непрерывным малым скачком [81-83]. В частном случае следует предположить, что изменение среднего размера капель и пузырей в зависимости от времени носит непрерывный характер и описывается уравнением изменения средней массы частиц по времени

$$
\frac{d m}{d t}= \pm \omega(a) m
$$

Здесь знак $(+)$ характерен для коалесценции, а знак $(-)$ - для дробления капель и пузырей. Экспериментальные исследования по дроблению и коалесценции капель и пузырей в турбулентном потоке подтверждают, что средний размер капель определяется на уровне минимального (дробления) или максимального (коалеценции), что характеризует агрегативную устойчивость дисперсной среды. С учетом изложенного в уравнении (32) $m$ рассматривается как приведенная масса относительно экстремальных значений капель и пузырей: $m=\frac{\pi}{6} \rho_{d}\left(a-a_{\min }\right)^{3}$ - для дробления и $m=\frac{\pi}{6} \rho_{d}\left(a_{\max }-a\right)^{3}$ - для коалесценции. В частном случае из уравнения (32) можно получить формулу, характеризующую изменение размеров капель при дроблении в виде

$$
\frac{d a}{d t}=-k(\omega, a)\left(a-a_{\min }\right)=f(a) ; \quad t=0, \quad a=a_{0}
$$

что является непрерывным процессом по времени, где $k(\omega, a)=\omega(a) / 3$. Экспериментально измеряемые средние размеры при дроблении капель в перемешивающих устройствах, где имеет место турбулентность потока, приведены в работах $[19,47,84]$. С использованием экспериментальных значений [47] в работах $[13,19]$ для частоты вращения мешалки $n=1000 \div 2500$ об/мин приведены следующие соотношения для определения начального и минимального размеров капель масла в водной среде: $a_{0}=1.75 \cdot 10^{2} \cdot\left(n \cdot d_{M}\right)^{-1.5}$ и $a_{\min }=1.695 \cdot 10^{4} \cdot\left(n \cdot d_{M}\right)^{-1.75}$ с использованием выражения $(14)$.

Сравнительный анализ расчетных данных и экспериментальных значений изменения среднего размера капель можно выполнить по информации, представленной на рис. 5 .

В силу того, что изменение размеров частиц можно рассматривать в виде непрерывной функции, уравнение Фоккера-Планка может быть записано так:

$$
\begin{gathered}
\frac{\partial P(a, t)}{\partial t}=-\frac{\partial}{\partial a}\left[P(a, t) \frac{d a}{d t}\right]+\frac{B}{2} \frac{\partial^{2} P(a, t)}{\partial a^{2}}, \\
t=0, \quad P(a, 0)=P_{0}(a) ; \quad a \rightarrow 0, \quad P(a, t) \rightarrow 0 .
\end{gathered}
$$


雨

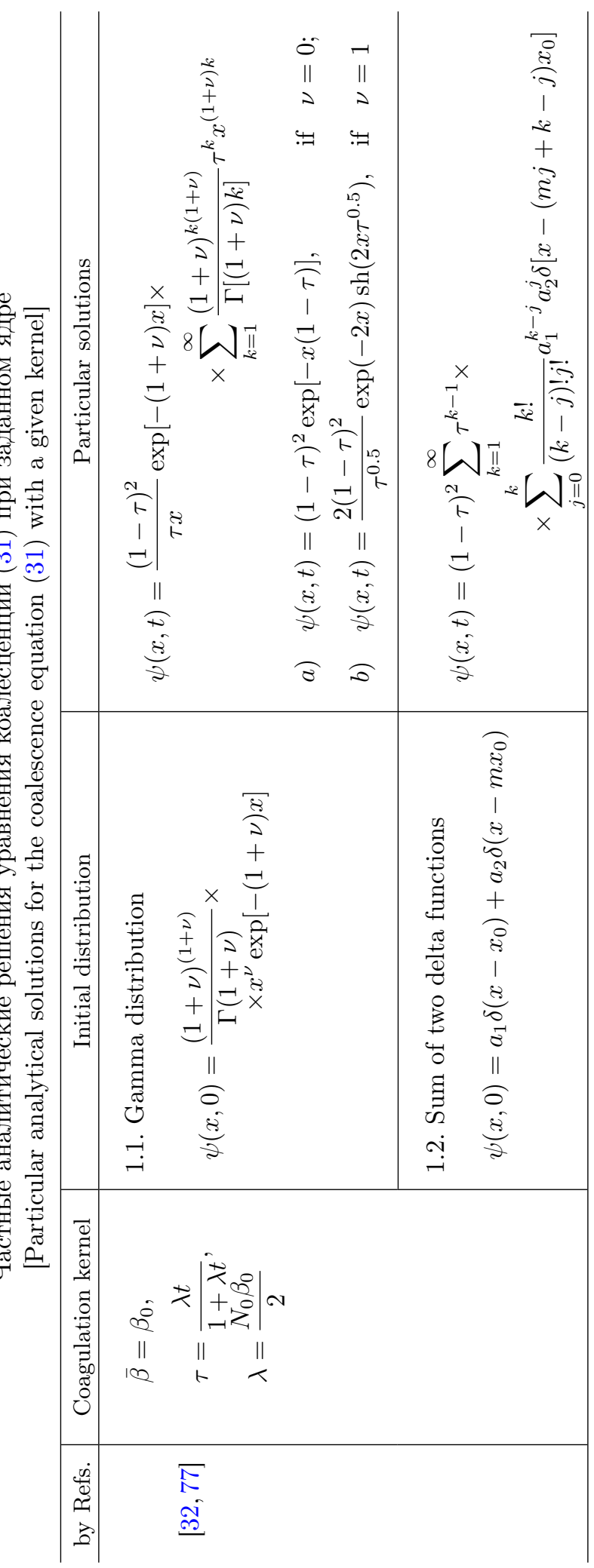




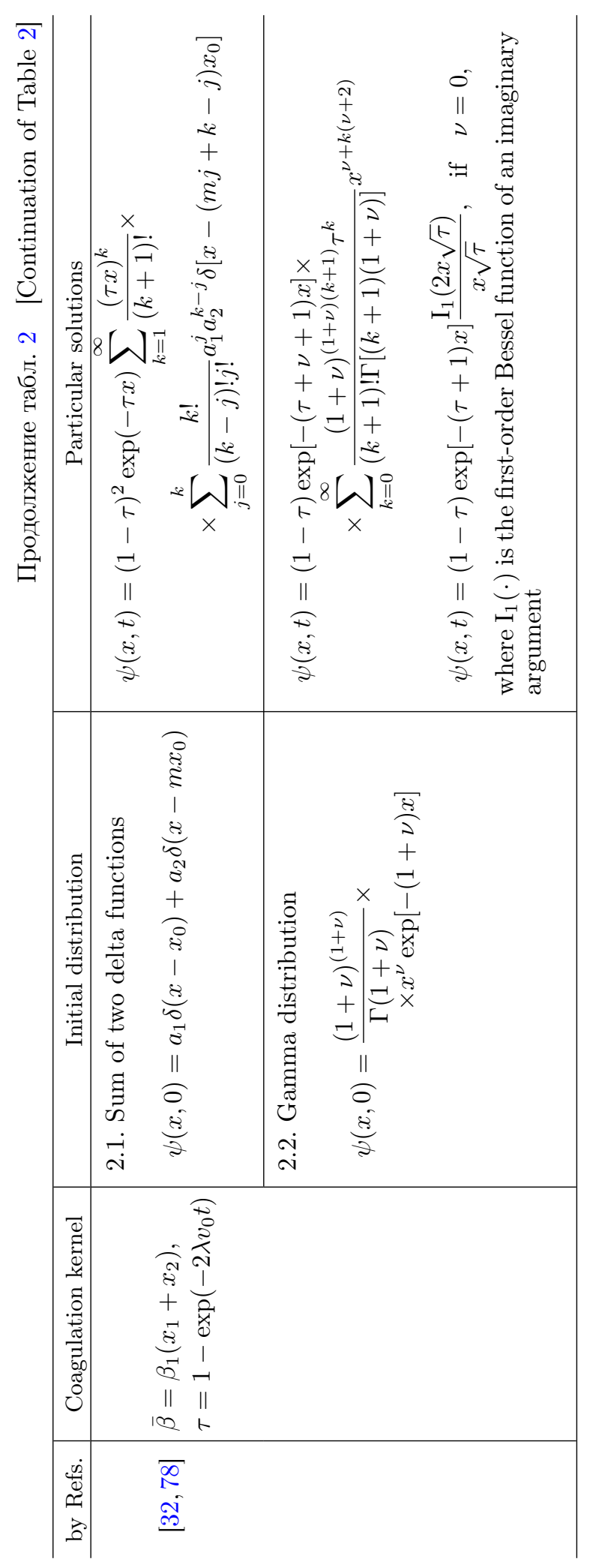




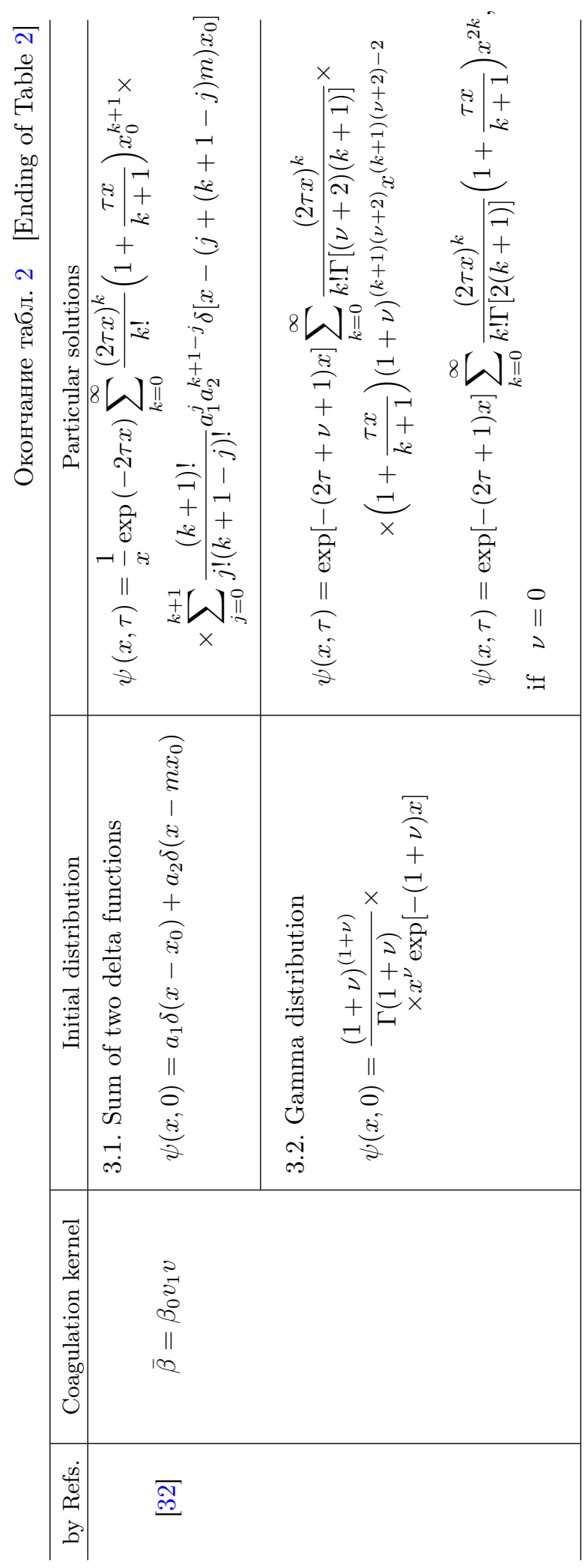




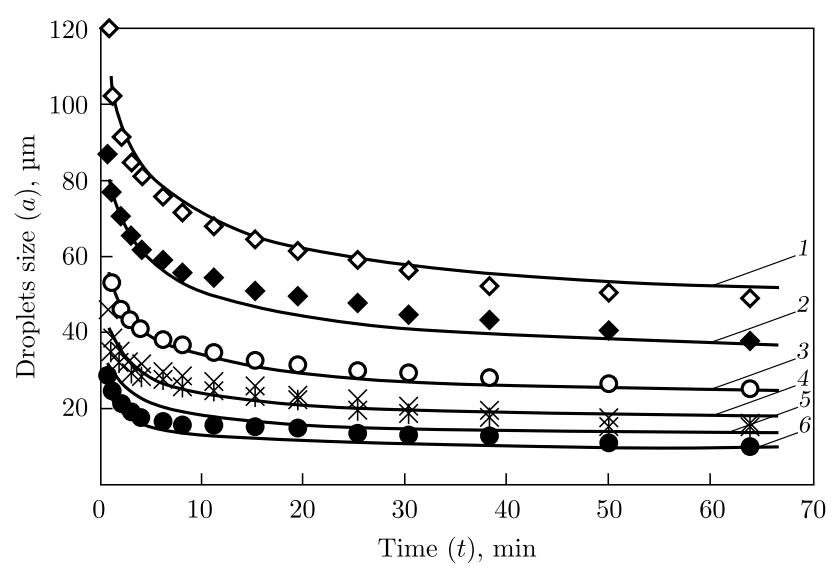

Рис. 5. Характер изменения среднего размера капель при различных частотах вращения мешалки: 1) $n=1000$ об/мин, 2) $n=1200$ об/мин, 3) $n=1500$ об/мин, 4) $n=1800$ об/мин, 5) $n=2100$ об/мин, 6) $n=2500$ об/мин

[Figure 5. The change in the average droplets size at different rotation frequency of a mixer: 1) $n=1000 \mathrm{rpm}, 2) n=1200 \mathrm{rpm}$, 3) $n=1500 \mathrm{rpm}, 4) n=1800 \mathrm{rpm}$, 5) $n=2100 \mathrm{rpm}, 6) n=2500 \mathrm{rpm}]$

Решение этого уравнения может представлять определенные трудности из-за вида функции $f(a)$ в (33). Некоторые частные аналитические решения уравнения (34) в зависимости от характера задания функции $f(a)$ можно найти в работах $[13,15,19,52,73,74]$ (табл. 3).

Стационарные решения уравнения Фоккера-Планка предложены в работе [73]. В некоторых случаях, например для мультимодальной экспериментальной функции распределения, решение (34) с использованием определенных эмпирических преобразований может быть представлено в виде суммы логнормальных распределений [19]:

$$
P(a, t)=\sum_{n=0}^{\infty} A_{n}(t) \exp \left[-m_{n}(t)\left(\ln a-\alpha_{s n}\right)^{2}\right]
$$

где $\alpha_{s n}$ - параметр, соответствующий логарифму максимального значения каждого экстремума. Экспериментальные исследования по коалесценции и дроблению капель и пузырей [32-34,47,72] показывают, что функция распределения носит мультимодальный характер, что объясняется наличием в потоке вторичных, третичных и т. д. явлений коалесценции и дробления. Причем число экстремумов в «хвосте» кривой распределения характеризует дополнительные акты коалесценции частиц, а число экстремумов в начале кривой распределения - число актов повторного дробления.

Обсуждение и анализ результатов. Теория и описание физических явлений коалесценции и дробления капель и пузырьков в изотропном турбулентном потоке представляет собой детерминированно-стохастическую проблему, включающую в себя решение задач детерминированного характера (гидродинамические задачи в турбулентном потоке) и описание процессов стохастической природы, связанных в основном со случайным скачкообразным изменением размеров частиц и их распределением по размерам и по 
m

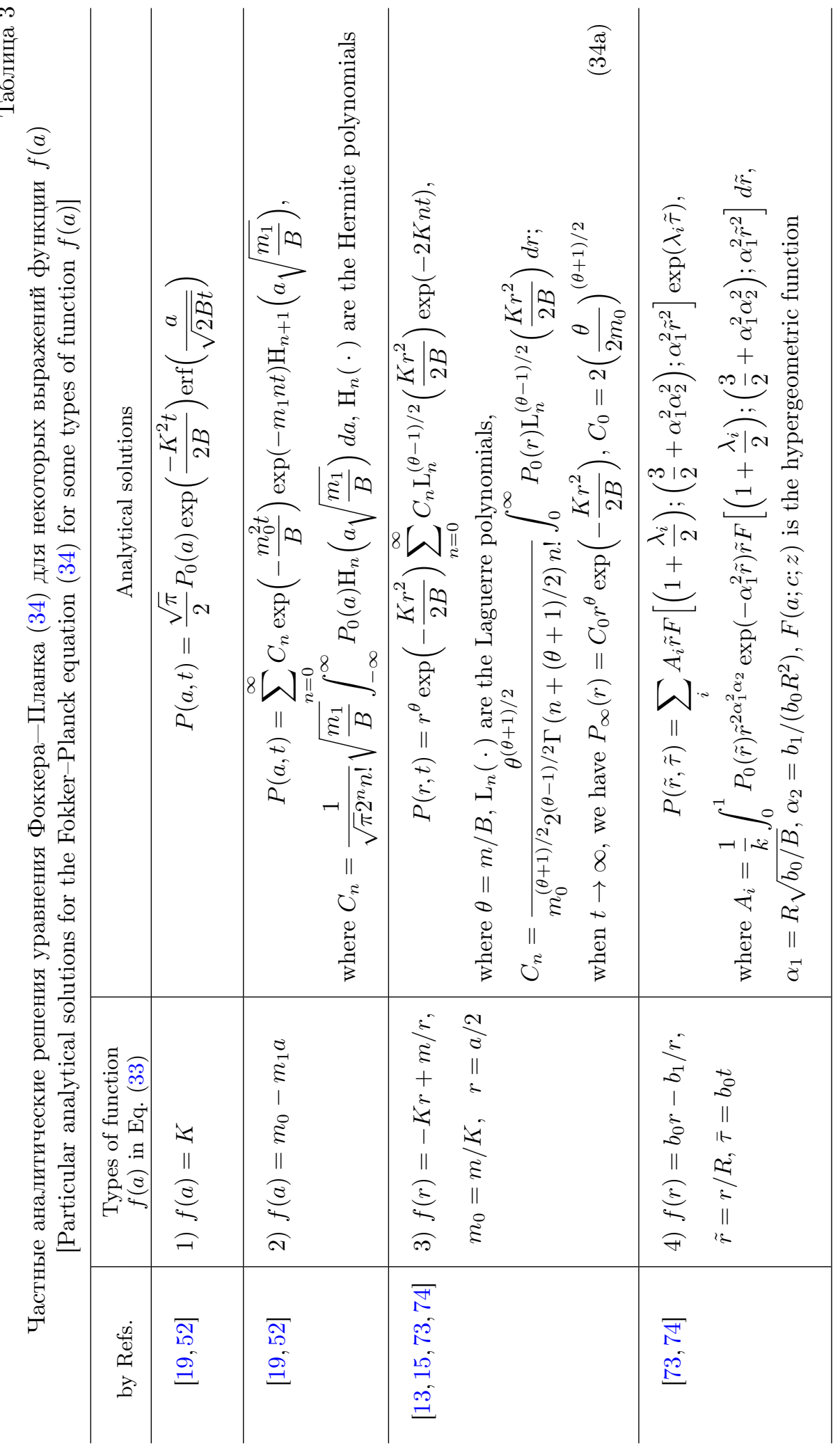


времени. В то же время проблемы коалесценции и дробления можно рассматривать с позиций теории «рождения и гибели» частиц [73] и теории катастроф [85], поскольку основу этих процессов составляет образование новых частиц, связанных с исчезновением или «гибелью» старых, появлением или «рождением» новых частиц, что характеризуется потерей агрегативной и седиментационной устойчивости дисперсной среды. Как следует из формул (2)-(12) и (17)-(19), важным параметром, обеспечивающим агрегативную устойчивость дисперсной среды типа «жидкость-жидкость» или «жидкость-газ» к дроблению, деформации и коалесценции, является коэффициент поверхностного натяжения, а в изотропном турбулентном потоке отношение $\sim \sigma / \varepsilon_{R}(15)$, поскольку эти параметры являются основными при оценке максимальных (2), (4)-(8) и минимальных (10)-(14) размеров капель, при вычислении частот дробления и коалесценции (17)-(20). В некоторых исследованиях значение максимального размера капель и пузырей связывают с критическим числом Вебера следующим образом: $a_{\max } \sim W \mathrm{We}_{\mathrm{cr}}^{0.6}$, где $\mathrm{We}_{\mathrm{cr}}=1.18$ [38], $\mathrm{We}_{\mathrm{cr}}=2.6$ [86], We $\mathrm{cr}=2.7 \div 7.8$ [87]. В большинстве случаев приведенные в литературе формулы для оценки минимальных и максимальных размеров капель и пузырей носят формальный характер, поскольку их сравнение с экспериментальными значениями дает большую ошибку, но при определенном подборе неизвестных коэффициентов (например, параметра $\gamma$ в уравнениях (4)-(8) и (9)-(14)) в этих формулах можно добиться удовлетворительной сходимости расчетных данных к экспериментальным в конкретных случаях.

В дисперсной среде возможны одновременное протекание процессов как коалесценции, так и дробления капель, причем в условиях равновесия между этими процессами наблюдается периодическое повторение этих явлений в зависимости от минимальных и максимальных размеров капель. Нарушение равновесия между явлениями коалесценции и дробления, связанное с изменением режима и внешних параметров, способствует смещению процесса в том или ином направлении. В частности, при достаточно больших значениях угловой скорости перемешивания процесс смещается в сторону как дробления, так и коалесценции, что связано с увеличением удельной диссипации энергии $\varepsilon_{R} \sim n^{3}$ и частоты дробления $\omega(a) \sim n^{0.5}[13,19]$. Увеличение скорости дробления капель способствует росту числа капель в единице объема и, соответственно, увеличению вероятности столкновения и укрупнения капель. Этот факт ярко проявляется в процессах жидкостной экстракции, расслоения и разделения эмульсий [88]. На практике явления коалесценции и дробления капель могут осложняться наличием различных пленок на поверхности капель, тормозящих протекание процесса. В частности, в системе «нефть-вода» на поверхности водных капель образуется защитная пленка за счет диффузии естественных ПАВ, содержащихся в сырой нефти на межфазной поверхности. Структурно-механическая устойчивость таких эмульсионных систем связана с образованием на границе раздела «нефть-вода» адсорбционных слоев, в состав которых входят асфальтены, парафины, смолы, минеральные соли и твердые частицы [89-91]. Анализ состава указанных оболочек на поверхности капель воды, находящейся в сырой нефти различных месторождений, подтверждает, что доминирующими стабилизаторами считаются асфальтены и смолы, в составе которых находятся высокоплавкие парафины и неорганические механические примеси. В связи с этим для коалесценции и дробления подобных систем прежде всего необходимо разрушение и разрыхление 
адсорбционного слоя различными поверхностно-активными веществами. Интенсификация подобных процессов разделения нефтяных эмульсий связана, прежде всего, с турбулизацией потока, поскольку высокочастотные пульсации турбулентности приводят к ослаблению адсорбционной пленки и межмолекулярных связей между ее основными компонентами, уменьшению прочности и разрыву пленки в результате их растяжения и сжатия, улучшению условий увеличения частоты столкновений.

Проблемы коалесценции и дробления капель, характеризующиеся сложным случайным скачкообразным поведением, таят в себе много сложностей и тонкостей. Более глубокий анализ этих явлений на основе математических закономерностей явлений переноса позволяют стандартным образом рассчитывать такие системы в некотором приближении как непрерывные с бесконечно малым скачком. Очевидно, что детерминированное описание этих явлений без учета их стохастической природы является неполным и может приводить к существенным отклонениям от истинной природы этих процессов. Использование кинетического уравнения коалесценции и дробления (28) и уравнения Фоккера-Планка (34) позволяет в широком смысле интерпретировать и анализировать эти явления в различные моменты времени. Следует отметить, что некоторые аналитические решения этих уравнений (табл. 2 и 3 ) позволяют получать важные теоретические результаты для исследования и анализа этих явлений. В частности, из решения уравнения Фоккера-Планка для асимптотического случая (34a) (табл. 3) следует, что при бесконечном времени протекания $(t \rightarrow \infty)$ конечное распределение частиц не зависит от значения и характера начального распределения. Причем при $\theta \rightarrow 0$ данное распределение совпадает с распределением Розена-Рамблера, используемого в работе [31], при $\theta=1-$ с распределением Релея, а при $\theta=2-$ с распределением Максвелла. Таким образом, этот вывод позволяет судить о стационарности функции распределения при большом времени протекания процессов коалесценции и дробления, инвариантных к заданию характера начального распределения. Вместе с тем многочисленные экспериментальные исследования [19,29-35,47] свидетельствуют о мультимодальности функции распределения, что объясняется наличием повторных актов коалесценции и дробления. Причем множество максимумов функции распределения в левой части соответствует повторным дроблениям капель, а в вибрирующей «хвостовой» части - повторной коалесценции. Для описания таких функций распределения наиболее приемлемым является выражение (35), представляющее собой суперпозицию множества логнормальных функций [19]. Следует отметить, что смещение спектров больших и малых капель носит практически взаимосвязанный характер. Однако через некоторое время, когда ресурсы крупнокапельного спектра исчерпываются, возможно, спектр начинает вести себя подобно одногорбовому распределению. Важно отметить, что на характер эволюции функции распределения и на коэффициент турбулентной диффузии существенное влияние оказывает выбывание частиц из рассмотренного объема в результате их всплытия или осаждения [37]. В этом случае спектр распределения существенно меняется с изменением скорости осаждения или всплытия. 


\section{Условные обозначения}

$a$

$B$

$C_{D}$

D

$D_{T}$

$D_{T P}$

$k_{R}$

$m$

$N$

$P$

$P(a, t)$

$R$

$R_{K}$

$t$

$\bar{U}$

$V^{\prime}$

$v$

$\bar{\beta}$

$\delta$

$\varepsilon_{R}$

$\eta_{c}, \eta_{d}$

$\lambda$

$\nu_{c}, \nu_{d}$

$\mu^{2}$

$\rho_{c}, \rho_{d}$

$\sigma$

$\theta$

$\vartheta$

$\varphi$

$\omega$

Mo

$\operatorname{Re}$

Sh

$\mathrm{Pe}$

We

размер частиц; тиц; фузии; фузии среды; фузии частиц;

масса частицы;

давление;

радиус частиц;

время; тока;

объем частицы; массы; частиц; частиц; тяжения;

число Мортона;

число Шервуда;

число Пекле;

число Вебера. коэффициент диффузии, имеющий стохастическую природу; коэффициент сопротивления час-

коэффициент молекулярной диф-

коэффициент турбулентной диф-

коэффициент турбулентной диф-

константа скорости дробления;

число частиц в единице объема;

функция распределения частиц;

радиус поверхности контакта;

среднеквадратичная скорость по-

пульсационная скорость турбулентного потока;

функция ядра коалесценции; толщина межфазной пленки; диссипация энергии в единице

динамическая вязкость среды и

масштаб турбулентности;

кинематическая вязкость среды и

собственные числа;

плотность среды и частиц;

коэффициент поверхностного на-

азимутальный угол;

коэффициент захвата;

объемная доля частиц;

частота дробления;

число Рейнольдса;

\section{Nomenclature}

size (diameter) of particles;

stochastic diffusion coefficient;

drag coefficient of particles;

coefficient of molecular diffusion;

coefficient of turbulent diffusion of a medium;

coefficient of turbulent diffusion of particles;

breakage rate constant;

particle mass;

is total number of particles per unit volume;

pressure;

particles distribution function;

particles radius;

contact surface radius;

time;

root mean square flow velocity;

pulsation velocity of the turbulent flow;

particles volume;

coalescence kernel function;

interfacial film thickness;

energy dissipation per unit mass;

dynamic viscosities of the medium and particles;

turbulence scale length;

kinematic viscosities of the medium and particles;

are the eigenvalues;

densities of the medium and particles;

surface tension coefficient;

azimuth angle;

capture ratio;

volume fraction of particles;

breakage frequency function;

Morton number;

Reynolds number;

Sherwood number;

Peclet number;

Weber number. 
Конкурирующие интересы. Мы не имеем конкурирующих интересов.

Авторский вклад и ответственность. Все авторы принимали участие в разработке концепции статьи и в написании рукописи. Авторы несут полную ответственность за предоставление окончательной рукописи в печать. Окончательная версия рукописи была одобрена всеми авторами.

Финансирование. Исследование выполнялось без финансирования.

Благодарность. Авторы благодарны рецензентам за тщательное прочтение статьи и ценные предложения и комментарии.

\section{Библиографический список}

1. Левич В. Г. Физико-химическая гидродинамика. М.: Физматлит, 1962.

2. Soo S. L. Fluid Dynamics of multiphase systems. Waltham, Mass.: Blasdell Publ., 1967.

3. Coulaloglou C. A., Tavlarides L. L. Description of interaction process in agitated liquidliquid dispersion// Chem. Eng. Sci., 1977. vol.32, no.11. pp. 1289-1297. doi: 10.1016/ 0009-2509 (77) 85023-9.

4. Prince M. J., Blanch H. W. Bubble coalescence and break-up in air-sparged bubble columns // AIChE J., 1990. vol.36, no. 10. pp. 1485-1499. doi: 10.1002/aic.690361004.

5. Hesketh R. P., Ethells A. W., Russell T. W. F. Bubble breakage in pipeline flow // Chem. Eng. Sci., 1991. vol. 46, no. 1. pp. 1-9. doi: 10.1016/0009-2509(91)80110-K.

6. Hesketh R. P., Ethells A. W., Russell T. W. F. Experimental observations of bubble breakage in turbulent flow // Ind. Eng. Chem. Res., 1991. vol.30, no. 5. pp. 835-841. doi: 10.1021/ ie00053a005.

7. Tsouris C., Tavlarides L. Breakage and coalescence models for drops in turbulent dispersions // AIChE J., 1994. vol.40, no. 3. pp. 395-406. doi: 10.1002/aic.690400303.

8. Luo H., Svendsen H. F. Theoretical model for drop and bubble breakup in turbulent dispersions // AIChE J., 1996. vol.42, no. 5. pp. 1225-1233. doi: 10.1002/aic.690420505.

9. Liu S., Li D. Drop coalescence in turbulent dispersions // Chem. Eng. Sci., 1999. vol.54, no. 23. pp. 5667-5675. doi: 10.1016/S0009-2509(99)00100-1.

10. Raymond F., Rozant J. M. A numerical and experimental study of the terminal velocity and shape of bubbles in viscous fluids // Chem. Eng. Sci., 2000. vol.55, no. 5. pp. 943-955. doi : 10.1016/S0009-2509(99)00385-1.

11. Galinat S., Masbernat O., Guiraud P., Daimazzonne C., Noik C. Drop break-up in turbulent pipe flow downstream of a restriction // Chem. Eng. Sci., 2005. vol. 60, no. 23. pp. 6511-6528. doi : 10.1016/j.ces.2005.05.012.

12. Ceylan S., Kelbaliyev G. Estimation of the maximum stable drop sizes, coalescence frequencies and the size distributions in isotropic turbulent dispersions // Colloid and Surfaces A: Physicochemical Engineering Aspects, 2003. vol.212, no.2-3. pp. 285-295. doi : 10.1016/S0927-7757(02)00326-6.

13. Келбалиев Г. И., Ибрагимов З. И. Коалесценция и дробление капель в изотропном турбулентном потоке // Теор. осн. хим. техн., 2009. Т. 43, № 3. С. 329-336.

14. Sarimeseli A., Kelbaliyev G. Modeling of the break-up of deformable particles in developed turbulent flow // Chem. Eng. Sci., 2004. vol. 59, no.6. pp. 1233-1240. doi: 10.1016/j.ces. 2003.09 .047$.

15. Келбалиев Г. И., Расулов С. Р. Гидродинамика и массоперенос в дисперсных средах. СПб.: Химиздат, 2014.

16. Blanchette F., Bigioni T. P. Dynamics of drop coalescence at fluid interfaces // J. Fluid Mech., 2009. vol.620. pp. 333-352. doi: 10.1017/S0022112008004801.

17. Narhe R., Beysens D., Nikolayev V. S. Dynamics of drop coalescence on a surface: The role of initial conditions and surface properties // Int. J. Thermophys., 2005. vol. 26, no. 6. pp. 1743-1757. doi : 10.1007/s10765-005-8593-4. 
18. Balmforth N. J., Llewellyn Smith S. G., Young W. R. Dynamics of interfaces and layers in a stratified turbulent fluid // J. Fluid Mech., 1998. vol.355. pp. 329-358. doi: 10.1017/ S0022112097007970.

19. Sis H., Kelbaliyev G., Chander S. Kinetics of drop breakage in stirred vessels under turbulent conditions // J. Dispersion Sci. and Technology, 2005. vol.26, no.5. pp. 565-573. doi: 10. 1081/DIS-200057638.

20. Liao Y., Lucas D. A literature review of theoretical models for drop and bubble breakup in turbulent dispersions // Chem. Eng. Sci., 2009. vol.64, no. 15. pp. 3389-3406. doi: 10. 1016/j.ces.2009.04.026.

21. Walter J. F., Blanch H. W. Bubble break-up in gas-liquid bioreactors: break-up in turbulent flows // Chem. Eng. J., 1986. vol. 32, no. 1. pp. B7-B17. doi: 10.1016/0300-9467 (86) 85011-0.

22. Narsimhan G. Model for drop coalescence in a locally isotropic turbulent flow field // J. Coll. Interf. Sci., 2004. vol.272, no. 1. pp. 197-209. doi: 10.1016/j.jcis.2003.11.057.

23. Wong D. C. Y., Simmons M. J. H., Decent S. P., Parau E. I., King A. C. Break-up dynamics and drop size distributions created from spiraling liquid jets // Intern. J. Multiphase Flow, 2004. vol. 30, no. 5. pp. 499-520. doi: 10.1016/j.ijmultiphaseflow.2004.04.002.

24. Kraume M., Gäbler A., Schulze K. Influence of physical properties on drop size distribution of stirred liquid-liquid dispersions // Chem. Eng. Tech., 2004. vol. 27, no. 3. pp. 330-334. doi: 10.1002/ceat. 200402006.

25. Revankar S. T. Coalescence and breakup of fluid particles in multi-phase flow / ICMF4 th International Conference on Multiphase Flow (New Orleans, Louisiana, USA, May 27June 1), 2001.

26. Vanni M. Approximate population balance equations for aggregation-breakage processes // J. Coll. Interf. Sci., 2000. vol.221, no. 2. pp. 143-160. doi: 10.1006/jcis.1999.6571.

27. Attarakih M. M., Bart H. J., Faqir N. M. Solution of the droplet breakage equation for interacting liquid-liquid dispersions: a conservative discretization approach // Chem. Eng. Sci., 2004. vol. 59, no. 12. pp. 2547-2565. doi: 10.1016/j.ces.2004.03.004.

28. Maniero R., Masbernat O., Climent E., Risso F. Modeling and simulation of inertial drop break-up in a turbulent pipe flow downstream of a restriction// Intern. J. Multiphase Flow, 2012. vol. 42. pp. 1-8. doi: 10.1016/j.ijmultiphaseflow. 2012.01.006.

29. Tobin T., Muralidhar R., Wright H., Ramkrishna D. Determination of coalescence frequencies in liquid-liquid dispersions: Effect of drop size dependence// Chem. Eng. Sci., 1990. vol. 45, no. 12. pp. 3491-3504. doi : 10.1016/0009-2509(90)87154-k.

30. Simmons M. J. H., Azzopardi B. J. Drop size distribution in dispersed liquid-liquid pipe flow// Intern. J. Multiphase Flow, 2001. vol.27, no.5. pp. 843-859. doi:10.1016/ s0301-9322(00)00055-0.

31. Angeli P., Hewitt O. F. Drop size distribution in horizontal oil-water dispersed flow // Chem. Eng. Sci., 2000. vol. 55, no. 16. pp. 3133-3143. doi: 10.1016/s0009-2509(99)00585-0.

32. Волощук В. М., Седунов Ю. С. Процессы коагуляиии в дисперсных системах. Л.: Гидрометеоиздат, 1975. [Voloshchuk V. M., Sedunov Yu. S. Protsessy koaguliatsii v dispersnykh sistemakh [Coagulation Processes in Dispersed Systems]. Leningrad: Gidrometeoizdat, 1975 (In Russian)].

33. Lasheras J., Martín-Bazán C., Montañés J. On the break-up of air bubble injected into fully developed turbulent flow. Part 1: Break-up frequency / 30th Fluid Dynamics Conference, 1999. doi: 10.2514/6.1999-3642.

34. Fei Y., Pang M. Bubble Coalescence and Breakup Phenomena: A Review // Recent Patents on Engineering, 2017. vol. 11, no. 2. pp. 80-88. doi: 10.2174/1872212111666170105115622.

35. Kostoglou M., Karabelas A. J. A contribution towards predicting the evolution of droplet size distribution in flowing dilute liquid/liquid dispersions // Chem. Eng. Sci., 2001. vol. 56, no. 14. pp. 4283-4292. doi: 10.1016/s0009-2509(01)00108-7.

36. Медников Е. П. Турбулентный перенос и осаждение аэрозолей. М.: Наука, 1982. 
37. Altunbas A., Kelbaliyev G., Ceylan K. Eddy diffusivity of particles in turbulent flow in rough cannels // J. Aerosol Sci., 2002. vol. 33, no. 7. pp. 1075-1086. doi : 10.1016/s0021-8502(02) $00054-\mathrm{x}$.

38. Hinze J. O. Fundamentals of the hydrodynamic mechanism of splitting in dispersion processes // AIChE J., 1955. vol.1, no.3. pp. 289-295. doi: 10.1002/aic.690010303.

39. Roccon A., De Paoli M., Zonta F., Soldati A. Viscosity-modulated breakup and coalescence of large drops in bounded turbulence // Phys. Rev. Fluids, 2017. vol. 2, no. 8, 083603. doi: 10.1103/physrevfluids.2.083603.

40. Baldyga J., Bourne J. R. Interpretation of turbulent mixing using fractals and multifractals // Chem. Eng. Sci., 1995. vol.50, no. 3. pp. 381-400. doi:10.1016/0009-2509(94) $00217-f$.

41. Qian D., McLaughlin J. B., Sankaranayanan K., Sundaresan S., Kontomaris K. Simulation of bubble breakup dynamics in homogeneous turbulence// Chem. Eng. Comm., 2006. vol. 193, no. 8. pp. 1038-1063. doi : 10.1080/00986440500354275.

42. Clift R., Grace J. R., Weber M. E. Bubbles, drops and particles. New York: Academic Press, 1978.

43. Kelbaliyev G., Ceylan K. Development of new empirical equations for estimation of drag coefficient, shape deformation and rising velocity gas bubbles or liquid drops // Chem. Eng. Comm., 2007. vol.194, no. 12. pp. 1623-1637. doi : 10.1080/00986440701446128.

44. Келбалиев Г. И. Коэффициент сопротивления твердых частиц, капель и пузырей различной формы // Теор. осн. хим. техн., 2011. Т. 45, № 3. С. 264-283.

45. Evans G. M., Jameson G. J., Atkinson B. W. Prediction of bubble size generated by a plunging liquids jet bubble column// Chem. Eng. Sci., 1992. vol.47, no.13-14. pp. 32653272. doi : 10.1016/0009-2509(92)85034-9.

46. Biń A. K. Gas entrainment by plunging liquid jets // Chem. Eng. Sci., 1993. vol. 48, no. 21. pp. 3585-3630. doi : 10.1016/0009-2509(93)81019-r.

47. Sis H., Chander S. Kinetics of emulsification of dodecane in the absence and presence of nonionic surfactants // Colloids and Surface A: Physicochemical Aspects, 2004. vol. 235, no. 1-3. pp. 113-120. doi: 10.1016/j.colsurfa.2004.01.001.

48. Pilch M., Erdman C. A. Use of breakup them data and velocity history data to predict the maximum size of stable fragments for acceleration-induced breakup of a liquid drop // Int. J. Multiphase Flow, 1987. vol. 13, no. 6. pp. 741-757. doi: 10.1016/0301-9322 (87)90063-2.

49. Castellano S., Sheibat-Othman N., Marchisio M., Buffo A., Charton S. Description of droplet coalescence and breakup in emulsions through a homogeneous population balance model // Chem. Eng. J., 2018. vol.354. pp. 1197-1207. doi: 10.1016/j.cej.2018.07.176.

50. Vankova N., Tcholakova S., Denkov N. D., Ivanov I. B., Vulchev V. D., Danner T. Emulsification in turbulent flow: 1 . Mean and maximum drop diameters in inertial and viscous regimes // J. Coll. Interf. Sci., 2007. vol.312, no.2. pp. 363-380. doi:10.1016/j.jcis. 2007.03 .059$.

51. Sleicher C. A. Maximum stable drop size in turbulent flow // AIChE J., 2004. vol. 8, no. 4. pp. 471-477. doi: 10.1002/aic.690080410.

52. Kelbaliyev G., Sarimeseli A. Modeling of drop coalescence in isotropic flow // J. Disp. Sci. Technol., 2006. vol.27, no. 4. pp. 443-451. doi: 10.1080/01932690500357305.

53. Yuan S., Fan Y., Li J., Cao Y. Influence of droplet coalescence and breakup on the separation process in wave-plate separators // Canad. J. Chem. Eng., 2018. vol. 96, no. 7. pp. 1627-1636. doi : 10.1002/cjce.23089.

54. Somwanshi P., Muralidhar K., Khandekar S. Influence of drop shape and coalescence on dropwise condensation over textured surfaces / Proceedings of the 15th International Heat Transfer Conference, IHTC-15, 2014.pp. 251-270. doi : 10.1615/ihtc15.kn.000013.

55. Hagesaether L., Jakobsen H. A., Svendsen H. F. A model for turbulent binary breakup of dispersed fluid particles // Chem. Eng. Sci., 2002. vol.57, no.16. pp. 3251-3267. doi: 10 . 1016/s0009-2509(02)00197-5. 
56. Wang T., Wang J., Jin Y. A novel theoretical breakup kernel function for bubbles/droplets in a turbulent flow // Chem. Eng. Sci., 2003. vol. 58, no. 20. pp. 4629-4637. doi: 10.1016/ j.ces.2003.07.009.

57. Chatzi E., Lee J. M. Analysis of interactions for liquid-liquid dispersions in agitated vessels // Ind. Eng. Chem. Res., 1987. vol.26, no.11. pp. 2263-2267. doi:10.1021/ ie00071a016.

58. Chatzi E., Kiparissides C. Dynamic simulation of bimodal drop size distributions in lowcoalescence batch dispersion systems // Chem. Eng. Sci., 1992. vol.47, no. 2. pp. 445-456. doi: 10.1016/0009-2509(92)80032-8.

59. Alopaeus V., Koskinen J., Keskinen K. I., Majander J. Simulation of the population balances for liquid-liquid systems in a nonideal stirred tank. Part 2: Parameter fitting and the use of the multiblock model for dense dispersions // Chem. Eng. Sci., 2002. vol. 57, no. 10. pp. 18151825. doi : 10.1016/s0009-2509(02)00067-2.

60. Lehr F., Milles M., Mewes D. Bubble-size distributions and flow fields in bubble columns // AIChE J., 2002. vol.48, no. 11. pp. 2426-2443. doi: 10.1002/aic.690481103.

61. Konno M., Aoki M., Saito S. Scale effect on breakup process in liquid-liquid agitated tanks // J. Chem. Eng. Japan, 1983. vol. 16, no.4. pp. 312-319. doi: 10.1252/jcej.16.312.

62. Bhaga D., Weber M. E. Bubbles in viscous liquids: shape, wakes and velocities // J. Fluid Mech., 1981. vol. 105. pp. 61-85. doi: 10.1017/s002211208100311x.

63. Броунштейн Б. И., Щеголев В. В. Гидродинамика, массообмен и теплообмен в колонных аппаратах. Л.: Химия, 1988.

64. Fanton X., Cazabat A. M., Quéré D. Thickness and shape of films driven by a Marangoni flow // Langmuir, 1996. vol. 12, no. 24. pp. 5875-5880. doi: 10.1021/1a960488a.

65. Leo L. Y., Matar O. K., Susana Pérez de Ortiz E., Hewitt G. F. A description of phase inversion behavior in agitated liquid-liquid dispersions under the Marangoni effect // Chem. Eng. Sci., 2002. vol. 57, no. 17. pp. 3505-3520. doi: 10.1016/s0009-2509(02)00260-9.

66. Scheludko A. Thin liquid film // Adv. Colloid Interf. Sci., 1967. vol. 1, no.4. pp. 391-464. doi : 10.1016/0001-8686(67)85001-2.

67. Chen J.-D., Slattery J. C. Effects of London-van der Waals forces on the thinning of a dimpled liquid films as a small drop or bubble approaches a horizontal solid phase // AIChE J., 1982. vol. 28, no.6. pp. 955-963. doi: 10.1002/aic.690280610.

68. Келбалиев Г. И., Сафаров Ф. Ф. исследование утончения межфазной пленки в процессах разделения нефтяных эмульсий // Химия и технология топлив и масел, 2011. № 4 . C. $18-23$.

69. Sherman Ph. Emulsion Science. London, New York: Academic Press, 1968.

70. Петров А. А., Блатова С. А. Изучение устойчивости углеводородных слоев на границе с водными растворами деэмульгаторов // Химия и технология топлив и масел, 1969. № 5. C. 25-32.

71. Burrill K. A., Woods D. R. Film shapes for deformable drops at liquid-liquid interfaces. II. The mechanisms of film drainage// J. Coll. Interf. Sci., 1973. vol.42, no. 1. pp. 15-34. doi : 10.1016/0021-9797(73)90004-0.

72. Lasheras J. C., Eastwood C., Martín-Bazán C., Montañés J. I. A review of statistical models for the break-up of an immiscible fluid immersed into a fully developed turbulent flow // Inter. J. Multiphase Flow, 2002. vol. 28, no. 2. pp. 247-278. doi: 10.1016/s0301-9322(01) 00046-5.

73. Gardiner C. W. Handbook of Stochastic Methods for Physics, Chemistry and the Natural Sciences/ Springer Series in Synergetics. New York: Springer, 1985. doi: 10.1007/ 978-3-662-02452-2.

74. Протодьяконов И. О., Богданов С. Р. Статистическая теория явлений переноса в прочессах химической технологии. Л.: Химия, 1983.

75. Melzak Z. A. A scalar transport equation // Trans. Amer. Math. Soc., 1957. vol. 85. pp. 547560. doi : 10.1090/S0002-9947-1957-0087880-6. 
76. Higashitani K., Yamanchi K., Matsuno Y., Hosokawa G. Turbulent coagulation of particles dispersed in a viscous fluid // J. Chem. Eng. Japan, 1983. vol. 16, no. 4. pp. 299-304. doi: 10. $1252 /$ jcej.16.299.

77. Келбалиев Г. И., Расулов С. Р., Мустафаева Г. Р. Моделирование явлений коалесценции капель в процессах разделения нефтяных эмульсий // Химия и технология топлив и масел, 2018. № 2. С. 24-28.

78. Головин А. М. K вопросу о решении уравнения коагуляции дождевых капель с учетом конденсации // Докл. АН СССР, 1963. Т. 148, №6. С. 1290-1293.

79. Alopaeus V., Laakkonen M., Aittamaa J. Solution of population balances with breakage and agglomeration by high-order moment-conserving method of classes // Chem. Eng. Sci., 2006. vol.61, no. 20. pp. 6732-6752. doi: 10.1016/j.ces.2006.07.010.

80. Maaß S., Wollny S., Sperling R., Kraume M. Numerical and experimental analysis of particle strain and breakage in turbulent dispersions // Chem. Eng. Res. Des., 2009. vol. 87, no. 4. pp. 565-572. doi: 10.1016/j.cherd.2009.01.002.

81. Аристов С. Н., Просвиряков Е. Ю. Об одном классе аналитических решений стационарной осесимметричной конвекции Бенара-Марангони вязкой несжимаемой жидкости // Вестн. Сам. гос. техн. ун-та. Сер. Физ.-мат. науки, 2013. №3(32). С. 110-118. doi: $10.14498 /$ vsgtu1205.

82. Vlasova S. S., Prosviryakov E. Yu. Two-dimensional convection of an incompressible viscous fluid with the heat exchange on the free border// Vestn. Samar. Gos. Tekhn. Univ., Ser. Fiz.-Mat. Nauki [J. Samara State Tech. Univ., Ser. Phys. Math. Sci.], 2016. vol. 20, no. 3. pp. 567-577. doi: 10.14498/vsgtu1483.

83. Privalova V. V., Prosviryakov E. Yu. Couette-Hiemenz exact solutions for the steady creeping convective flow of a viscous incompressible fluid, with allowance made for heat recovery // Vestn. Samar. Gos. Tekhn. Univ., Ser. Fiz.-Mat. Nauki [J. Samara State Tech. Univ., Ser. Phys. Math. Sci.], 2018. vol. 22, no. 3. pp. 532-548. doi: 10.14498/vsgtu1638.

84. Sajjadi S., Zerfa M., Brooks B. W. Dynamic behaviour of drops in oil/water/oil dispersions // Chem. Eng. Sci., 2002. vol.57, no.4. pp. 663-675. doi: 10.1016/S0009-2509(01) 00415-8.

85. Poston T., Stewart I. Catastrophe theory and its applications / Surveys and Reference Works in Mathematics. vol. 2. London: Pitman, 1978. xviii+491 pp.

86. Sevik M., Park S. H. The splitting of drops and bubbles by turbulent fluid flow // J. Fluids Eng., 1973. vol.95, no. 1. pp. 53-60. doi: 10.1115/1.3446958.

87. Risso F., Farbe J. Oscillations and breakup of a bubble immersed in a turbulent field // J. Fluid Mech., 1998. vol.372. pp. 323-335. doi: 10.1017/S0022112098002705.

88. Келбалиев Г. И., Сулейманов Г. З., Зорофи Ф. А., Гасанов А. А.,Рустамова А. И. Экстракционное разделение и очистка сточных вод органическими растворителями с рециркуляцией// Химическая промышленность, 2011. Т.88, №1. С. 35-41; Kelbaliyev G. I., Suleimanov G. Z., Zorofi P. A., Gasanov A. A., Rustamova A. I. Extraction separation and cleaning of sewage waters by organic solvents with recirculation// Russ. J. Appl. Chem., 2011. vol. 84, no.6. pp. 1114-1119. doi: 10.1134/S1070427211060383.

89. Sjöblom J., Urdahl O., Høiland H., Christy A. A., Johansen E. J. Water-in-crude oil emulsions. Formation, characterization, and destabilization / Surfactants and Macromolecules: Self-Assembly at Interfaces and in Bulk/ Progress in Colloid and Polymer Science, 82. Darmstadt: Steinkopff, 1990. pp. 131-139. doi : 10.1007/BFb0118251.

90. Позднышев Г. Н. Стабилизачия и разрушение нефтяных эмульсий. М.: Недра, 1982.

91. Тронов В. П. Разрушение эмульсий при добыче нефти. М.: Недра, 1974. 
MSC: 76F05, 76V05

\title{
Mathematical modeling of coalescence and breakage of droplets and bubbles in an isotropic turbulent flow: A review
}

\author{
G. I. Kelbaliyev ${ }^{1}$, S. R. Rasulov ${ }^{2}$ \\ 1 Institute of Catalysis and Inorganic Chemistry, \\ Azerbaijan National Academy of Sciences, \\ 29, Javid Ave, Baku, AZ 1143, Azerbaijan. \\ 2 Azerbaijan State University of Oil and Industry, \\ 34, Azadlig Ave, Baku, AZ 1010, Azerbaijan.
}

\begin{abstract}
This review devoted to the theoretical analysis, calculation, and modeling of the processes of merging and breakage of droplets and bubbles in an isotropic turbulent flow. We have analyzed a number of studies on these issues. The problems of determining the minimum and maximum sizes of droplets and bubbles, as well as breakage and merging frequencies, which are associated with the solution of the diffusion equation of mass transfer, are considered. The merging of droplets is considered as a result of the thinning of the interfacial film formed by two drops as a result of their collision. A mathematical description of the refinement of the interfacial film, taking into account the Marangoni effect, is proposed. Analysis of many studies, including our own, showed that, depending on the scale of turbulent pulsations, the extreme size, as well as the frequencies of coalescence and breakage of droplets and bubbles, depend on the specific dissipation energy in the turbulent flow, on their sizes and on the physical properties of the particles and the medium. Important parameters that provide aggregative stability of a liquid-liquid or liquid-gas type dispersion medium to breakage, deformation and fusion are the surface tension coefficient and energy dissipation, the physical properties of the medium and particles, and in an isotropic turbulent flow the ratio of the surface coefficient tension to specific energy dissipation.

Problems related to the evolution of the particle distribution function in time and size under isotropic turbulence using solutions of the FokkerPlanck stochastic equation for continuous variation of the sizes of droplets
\end{abstract}

\section{Review Article}

(우(;) The content is published under the terms of the Creative Commons Attribution 4.0 International License (http://creativecommons.org/licenses/by/4.0/)

Please cite this article in press as:

Kelbaliyev G. I., Rasulov S. R. Mathematical modeling of coalescence and breakage of droplets and bubbles in an isotropic turbulent flow: A review, Vestn. Samar. Gos. Tekhn. Univ., Ser. Fiz.-Mat. Nauki [J. Samara State Tech. Univ., Ser. Phys. Math. Sci.], 2019, vol. 23, no. 3, pp. 541-581. doi: 10.14498/vsgtu1664 (In Russian).

\section{Authors' Details:}

Gudret I. Kelbaliyev (D) https://orcid.org/0000-0002-6275-3732

Dr. Techn. Sci.; Corresponding member of Academy of Sciences of Azerbaijan; Head of Division; Div. of Modeling and Technology of Chemical and Ecological Processes;

e-mail: kudret.kelbaliev@mail.ru

Sakit R. Rasulov (1) https://orcid.org/0000-0002-1548-3143

Dr. Techn. Sci., Professor; Head of Dept.; Dept. of Industrial Safety and Labor Protection; e-mail: rasulovsakit@gmail.com 
and bubbles and the integro-differential kinetic equation of coalescence and fragmentation for jump-like changes in particle sizes are also considered. A set of analytical solutions of these equations for particular cases is proposed. A more in-depth analysis based on the mathematical laws of the transport phenomena makes it possible in the standard way to calculate such systems in an approximation, such as continuous, with an infinitely small jump. It is shown that the deterministic description of these phenomena without taking into account their stochastic nature is incomplete and can lead to significant deviations from the true nature of the above processes.

The results obtained are compared with the existing experimental data on coalescence and breakage of droplets and bubbles, which showed satisfactory agreement with the calculated values.

Keywords: mass transfer, interfacial film, energy dissipation, isotropic turbulence, surface tension, aggregative stability.

Received: $14^{\text {th }}$ December, $2018 /$ Revised: $7^{\text {th }}$ March, $2019 /$ Accepted: $10^{\text {th }}$ June, $2019 /$ First online: $22^{\text {nd }}$ October, 2019

Competing interests. We have no competing interests.

Authors' contributions and responsibilities. Each author has participated in the article concept development and in the manuscript writing. The authors are absolutely responsible for submitting the final manuscript in print. Each author has approved the final version of manuscript.

Funding. The research has not had any funding.

Acknowledgments. The authors are grateful to the reviewers for careful reading of the paper and valuable suggestions and comments.

\section{References}

1. Levich V. G. Physicochemical hydrodynamics, Prentice-Hall international series in the physical and chemical engineering sciences. Englewood Cliffs, N.J., Prentice-Hall, 1962.

2. Soo S. L. Fluid Dynamics of multiphase systems. Waltham, Mass., Blasdell Publ., 1967.

3. Coulaloglou C. A., Tavlarides L. L. Description of interaction process in agitated liquidliquid dispersion, Chem. Eng. Sci., 1977, vol.32, no.11, pp. 1289-1297. doi:10.1016/ 0009-2509 (77) 85023-9.

4. Prince M. J., Blanch H. W. Bubble coalescence and break-up in air-sparged bubble columns, AIChE J., 1990, vol.36, no. 10, pp. 1485-1499. doi: 10.1002/aic.690361004.

5. Hesketh R. P., Ethells A. W., Russell T. W. F. Bubble breakage in pipeline flow, Chem. Eng. Sci., 1991, vol. 46, no. 1, pp. 1-9. doi : 10.1016/0009-2509(91)80110-K.

6. Hesketh R. P., Ethells A. W., Russell T. W. F. Experimental observations of bubble breakage in turbulent flow, Ind. Eng. Chem. Res., 1991, vol.30, no.5, pp. 835-841. doi: 10.1021/ ie00053a005.

7. Tsouris C., Tavlarides L. Breakage and coalescence models for drops in turbulent dispersions, AIChE J., 1994, vol.40, no. 3, pp. 395-406. doi: 10.1002/aic.690400303.

8. Luo H., Svendsen H. F. Theoretical model for drop and bubble breakup in turbulent dispersions, AIChE J., 1996, vol.42, no.5, pp. 1225-1233. doi: 10.1002/aic.690420505.

9. Liu S., Li D. Drop coalescence in turbulent dispersions, Chem. Eng. Sci., 1999, vol.54, no. 23, pp. 5667-5675. doi: 10.1016/S0009-2509(99)00100-1.

10. Raymond F., Rozant J. M. A numerical and experimental study of the terminal velocity and shape of bubbles in viscous fluids, Chem. Eng. Sci., 2000, vol.55, no. 5, pp. 943-955. doi: 10.1016/S0009-2509(99)00385-1. 
11. Galinat S., Masbernat O., Guiraud P., Daimazzonne C., Noik C. Drop break-up in turbulent pipe flow downstream of a restriction, Chem. Eng. Sci., 2005, vol. 60, no. 23, pp. 6511-6528. doi: $10.1016 / j$.ces.2005.05.012.

12. Ceylan S., Kelbaliyev G. Estimation of the maximum stable drop sizes, coalescence frequencies and the size distributions in isotropic turbulent dispersions, Colloid and Surfaces A: Physicochemical Engineering Aspects, 2003, vol.212, no.2-3, pp. 285-295. doi:10.1016/ S0927-7757 (02) 00326-6.

13. Kelbaliev G. I., Ibragimov Z. I. Coalescence and fragmentation of drops in an isotropic turbulent flow, Theor. Found. Chem. Eng., 2009, vol.43, no. 3, pp. 314-320. doi: 10.1134/ S0040579509030117.

14. Sarimeseli A., Kelbaliyev G. Modeling of the break-up of deformable particles in developed turbulent flow, Chem. Eng. Sci., 2004, vol.59, no.6, pp. 1233-1240. doi: 10.1016/j.ces. 2003.09 .047$.

15. Kelbaliyev G. I., Rasulov S. R. Gidrodinamika i massoperenos v dispersnykh sredakh [Hydrodynamics and Mass Transfer in Dispersed Media]. St. Petersburg, Khimizdat, 2014 (In Russian).

16. Blanchette F., Bigioni T. P. Dynamics of drop coalescence at fluid interfaces, J. Fluid Mech., 2009, vol. 620, pp. 333-352. doi: 10.1017/S0022112008004801.

17. Narhe R., Beysens D., Nikolayev V. S. Dynamics of drop coalescence on a surface: The role of initial conditions and surface properties, Int. J. Thermophys., 2005, vol. 26, no. 6, pp. 1743-1757. doi: 10.1007/s10765-005-8593-4.

18. Balmforth N. J., Llewellyn Smith S. G., Young W. R. Dynamics of interfaces and layers in a stratified turbulent fluid, J. Fluid Mech., 1998, vol. 355, pp. 329-358. doi: 10.1017/ S0022112097007970.

19. Sis H., Kelbaliyev G., Chander S. Kinetics of drop breakage in stirred vessels under turbulent conditions, J. Dispersion Sci. and Technology, 2005, vol.26, no. 5, pp. 565-573. doi: 10. 1081/DIS-200057638.

20. Liao Y., Lucas D. A literature review of theoretical models for drop and bubble breakup in turbulent dispersions, Chem. Eng. Sci., 2009, vol.64, no. 15, pp. 3389-3406. doi: 10.1016/ j.ces.2009.04.026.

21. Walter J. F., Blanch H. W. Bubble break-up in gas-liquid bioreactors: break-up in turbulent flows, Chem. Eng. J., 1986, vol.32, no.1, pp. B7-B17. doi: 10.1016/0300-9467(86) 85011-0.

22. Narsimhan G. Model for drop coalescence in a locally isotropic turbulent flow field, J. Coll. Interf. Sci., 2004, vol. 272, no. 1, pp. 197-209. doi: 10.1016/j.jcis.2003.11.057.

23. Wong D. C. Y., Simmons M. J. H., Decent S. P., Parau E. I., King A. C. Break-up dynamics and drop size distributions created from spiraling liquid jets, Intern. J. Multiphase Flow, 2004, vol. 30, no. 5, pp. 499-520. doi: 10.1016/j.ijmultiphaseflow.2004.04.002.

24. Kraume M., Gäbler A., Schulze K. Influence of physical properties on drop size distribution of stirred liquid-liquid dispersions, Chem. Eng. Tech., 2004, vol. 27, no. 3, pp. 330-334. doi : 10.1002/ceat. 200402006 .

25. Revankar S. T. Coalescence and breakup of fluid particles in multi-phase flow, In: ICMF4 th International Conference on Multiphase Flow (New Orleans, Louisiana, USA, May 27June 1), 2001.

26. Vanni M. Approximate population balance equations for aggregation-breakage processes, J. Coll. Interf. Sci., 2000, vol.221, no. 2, pp. 143-160. doi: 10.1006/jcis.1999.6571.

27. Attarakih M. M., Bart H. J., Faqir N. M. Solution of the droplet breakage equation for interacting liquid-liquid dispersions: a conservative discretization approach, Chem. Eng. Sci., 2004, vol. 59, no. 12, pp. 2547-2565. doi: 10.1016/j.ces.2004.03.004.

28. Maniero R., Masbernat O., Climent E., Risso F. Modeling and simulation of inertial drop break-up in a turbulent pipe flow downstream of a restriction, Intern. J. Multiphase Flow, 2012, vol. 42, pp. 1-8. doi : 10.1016/j.ijmultiphaseflow.2012.01.006. 
29. Tobin T., Muralidhar R., Wright H., Ramkrishna D. Determination of coalescence frequencies in liquid-liquid dispersions: Effect of drop size dependence, Chem. Eng. Sci., 1990, vol. 45, no. 12, pp. 3491-3504. doi : 10.1016/0009-2509(90)87154-k.

30. Simmons M. J. H., Azzopardi B. J. Drop size distribution in dispersed liquid-liquid pipe flow, Intern. J. Multiphase Flow, 2001, vol. 27, no. 5, pp. 843-859. doi : 10.1016/s0301-9322 (00) 00055- 0 .

31. Angeli P., Hewitt O. F. Drop size distribution in horizontal oil-water dispersed flow, Chem. Eng. Sci., 2000, vol. 55, no. 16, pp. 3133-3143. doi: 10.1016/s0009-2509(99)00585-0.

32. Voloshchuk V. M., Sedunov Yu. S. Protsessy koaguliatsii v dispersnykh sistemakh [Coagulation Processes in Dispersed Systems]. Leningrad, Gidrometeoizdat, 1975 (In Russian).

33. Lasheras J., Martín-Bazán C., Montañés J. On the break-up of air bubble injected into fully developed turbulent flow. Part 1: Break-up frequency, In: 30th Fluid Dynamics Conference, 1999. doi : 10.2514/6.1999-3642.

34. Fei Y., Pang M. Bubble Coalescence and Breakup Phenomena: A Review, Recent Patents on Engineering, 2017, vol.11, no. 2, pp. 80-88. doi: 10.2174/1872212111666170105115622.

35. Kostoglou M., Karabelas A. J. A contribution towards predicting the evolution of droplet size distribution in flowing dilute liquid/liquid dispersions, Chem. Eng. Sci., 2001, vol. 56, no. 14, pp. 4283-4292. doi: 10.1016/s0009-2509(01)00108-7.

36. Mednikov E. P. Turbulentnyi perenos i osazhdenie aerozolei [Turbulent Transfer and Deposition of Aerosols]. Moscow, Nauka, 1982 (In Russian).

37. Altunbas A., Kelbaliyev G., Ceylan K. Eddy diffusivity of particles in turbulent flow in rough cannels, J. Aerosol Sci., 2002, vol.33, no. 7, pp. 1075-1086. doi: 10.1016/s0021-8502(02) $00054-\mathrm{x}$.

38. Hinze J. O. Fundamentals of the hydrodynamic mechanism of splitting in dispersion processes, AIChE J., 1955, vol.1, no. 3, pp. 289-295. doi: 10.1002/aic.690010303.

39. Roccon A., De Paoli M., Zonta F., Soldati A. Viscosity-modulated breakup and coalescence of large drops in bounded turbulence, Phys. Rev. Fluids, 2017, vol. 2, no. 8, 083603. doi : 10 . 1103/physrevfluids.2.083603.

40. Baldyga J., Bourne J. R. Interpretation of turbulent mixing using fractals and multifractals, Chem. Eng. Sci., 1995, vol. 50, no. 3, pp. 381-400. doi: 10.1016/0009-2509(94)00217-f.

41. Qian D., McLaughlin J. B., Sankaranayanan K., Sundaresan S., Kontomaris K. Simulation of bubble breakup dynamics in homogeneous turbulence, Chem. Eng. Comm., 2006, vol. 193, no. 8, pp. 1038-1063. doi : 10.1080/00986440500354275.

42. Clift R., Grace J. R., Weber M. E. Bubbles, drops and particles. New York, Academic Press, 1978.

43. Kelbaliyev G., Ceylan K. Development of new empirical equations for estimation of drag coefficient, shape deformation and rising velocity gas bubbles or liquid drops, Chem. Eng. Comm., 2007, vol. 194, no. 12, pp. 1623-1637. doi : 10.1080/00986440701446128.

44. Kelbaliyev G. I. Drag coefficients of variously shaped solid particles, drops, and bubbles, Theor. Found. Chem. Eng., 2011, vol.45, no.3, pp. 248-266. doi:10.1134/ S0040579511020084.

45. Evans G. M., Jameson G. J., Atkinson B. W. Prediction of bubble size generated by a plunging liquids jet bubble column, Chem. Eng. Sci., 1992, vol.47, no.13-14, pp. 32653272. doi : 10.1016/0009-2509(92)85034-9.

46. Biń A. K. Gas entrainment by plunging liquid jets, Chem. Eng. Sci., 1993, vol.48, no. 21, pp. 3585-3630. doi: 10.1016/0009-2509(93)81019-r.

47. Sis H., Chander S. Kinetics of emulsification of dodecane in the absence and presence of nonionic surfactants, Colloids and Surface A: Physicochemical Aspects, 2004, vol. 235, no. 13, pp. 113-120. doi: 10.1016/j.colsurfa.2004.01.001.

48. Pilch M., Erdman C. A. Use of breakup them data and velocity history data to predict the maximum size of stable fragments for acceleration-induced breakup of a liquid drop, Int. J. Multiphase Flow, 1987, vol. 13, no. 6, pp. 741-757. doi : 10.1016/0301-9322 (87)90063-2. 
49. Castellano S., Sheibat-Othman N., Marchisio M., Buffo A., Charton S. Description of droplet coalescence and breakup in emulsions through a homogeneous population balance model, Chem. Eng. J., 2018, vol. 354, pp. 1197-1207. doi: 10.1016/j.cej.2018.07.176.

50. Vankova N., Tcholakova S., Denkov N. D., Ivanov I. B., Vulchev V. D., Danner T. Emulsification in turbulent flow: 1 . Mean and maximum drop diameters in inertial and viscous regimes, J. Coll. Interf. Sci., 2007, vol.312, no.2, pp. 363-380. doi: 10.1016/j.jcis.2007.03.059.

51. Sleicher C. A. Maximum stable drop size in turbulent flow, AIChE J., 2004, vol. 8, no. 4, pp. 471-477. doi: 10.1002/aic.690080410.

52. Kelbaliyev G., Sarimeseli A. Modeling of drop coalescence in isotropic flow, J. Disp. Sci. Technol., 2006, vol.27, no.4, pp. 443-451. doi: 10.1080/01932690500357305.

53. Yuan S., Fan Y., Li J., Cao Y. Influence of droplet coalescence and breakup on the separation process in wave-plate separators, Canad. J. Chem. Eng., 2018, vol. 96, no. 7, pp. 1627-1636. doi: 10.1002/cjce.23089.

54. Somwanshi P., Muralidhar K., Khandekar S. Influence of drop shape and coalescence on dropwise condensation over textured surfaces, In: Proceedings of the 15th International Heat Transfer Conference, IHTC-15, 2014, pp. 251-270. doi: 10.1615/ihtc15.kn.000013.

55. Hagesaether L., Jakobsen H. A., Svendsen H. F. A model for turbulent binary breakup of dispersed fluid particles, Chem. Eng. Sci., 2002, vol.57, no. 16, pp. 3251-3267. doi: 10 . 1016/s0009-2509(02)00197-5.

56. Wang T., Wang J., Jin Y. A novel theoretical breakup kernel function for bubbles/droplets in a turbulent flow, Chem. Eng. Sci., 2003, vol. 58, no. 20, pp. 4629-4637. doi: 10.1016/j. ces.2003.07.009.

57. Chatzi E., Lee J. M. Analysis of interactions for liquid-liquid dispersions in agitated vessels, Ind. Eng. Chem. Res., 1987, vol. 26, no. 11, pp. 2263-2267. doi: 10.1021/ie00071a016.

58. Chatzi E., Kiparissides C. Dynamic simulation of bimodal drop size distributions in lowcoalescence batch dispersion systems, Chem. Eng. Sci., 1992, vol.47, no. 2, pp. 445-456. doi : 10.1016/0009-2509(92)80032-8.

59. Alopaeus V., Koskinen J., Keskinen K. I., Majander J. Simulation of the population balances for liquid-liquid systems in a nonideal stirred tank. Part 2: Parameter fitting and the use of the multiblock model for dense dispersions, Chem. Eng. Sci., 2002, vol. 57, no. 10, pp. 18151825. doi : 10.1016/s0009-2509(02)00067-2.

60. Lehr F., Milles M., Mewes D. Bubble-size distributions and flow fields in bubble columns, AIChE J., 2002, vol.48, no.11, pp. 2426-2443. doi: 10.1002/aic.690481103.

61. Konno M., Aoki M., Saito S. Scale effect on breakup process in liquid-liquid agitated tanks, J. Chem. Eng. Japan, 1983, vol. 16, no. 4, pp. 312-319. doi: 10.1252/jcej.16.312.

62. Bhaga D., Weber M. E. Bubbles in viscous liquids: shape, wakes and velocities, J. Fluid Mech., 1981, vol. 105, pp. 61-85. doi: 10.1017/s002211208100311x.

63. Brounstein B. I., Shchegolev V. V. Gidrodinamika, massoobmen i teploobmen $v$ kolonnykh apparatakh [Hydrodynamics, Mass Transfer and Heat Transfer in Column Apparatus]. Leningrad, Khimiya, 1988 (In Russian).

64. Fanton X., Cazabat A. M., Quéré D. Thickness and shape of films driven by a Marangoni flow, Langmuir, 1996, vol.12, no. 24, pp. 5875-5880. doi: 10.1021/la960488a.

65. Leo L. Y., Matar O. K., Susana Pérez de Ortiz E., Hewitt G. F. A description of phase inversion behavior in agitated liquid-liquid dispersions under the Marangoni effect, Chem. Eng. Sci., 2002, vol. 57, no. 17, pp. 3505-3520. doi: 10.1016/s0009-2509(02)00260-9.

66. Scheludko A. Thin liquid film, Adv. Colloid Interf. Sci., 1967, vol.1, no.4, pp. 391-464. doi : 10.1016/0001-8686(67)85001-2.

67. Chen J.-D., Slattery J. C. Effects of London-van der Waals forces on the thinning of a dimpled liquid films as a small drop or bubble approaches a horizontal solid phase, AIChE J., 1982, vol.28, no. 6, pp. 955-963. doi: 10.1002/aic.690280610.

68. Kelbaliev G. I., Safarov F. F. Study of interphase film thinning in petroleum emulsion separation processes, Chem. Technol. Fuels Oils, 2011, vol. 47, no.4, pp. 268-277. doi: 10. 1007/s10553-011-0295-y. 
69. Sherman Ph. Emulsion Science. London, New York, Academic Press, 1968.

70. Petrov A. A., Blatova S. A. An investigation of the stability of hydrocarbon layers at the boundary with aqueous solutions of de-emulsifiers, Chem. Technol. Fuels Oils, 1969, vol.5, no. 5, pp. 343-350. doi: 10.1007/bf00716035.

71. Burrill K. A., Woods D. R. Film shapes for deformable drops at liquid-liquid interfaces. II. The mechanisms of film drainage, J. Coll. Interf. Sci., 1973, vol.42, no. 1, pp. 15-34. doi : 10.1016/0021-9797(73) 90004-0.

72. Lasheras J. C., Eastwood C., Martín-Bazán C., Montañés J. I. A review of statistical models for the break-up of an immiscible fluid immersed into a fully developed turbulent flow, Inter. J. Multiphase Flow, 2002, vol. 28, no. 2, pp. 247-278. doi: 10.1016/s0301-9322(01) 00046-5.

73. Gardiner C. W. Handbook of Stochastic Methods for Physics, Chemistry and the Natural Sciences, Springer Series in Synergetics. New York, Springer, 1985. doi: 10.1007/ 978-3-662-02452-2.

74. Protodyakonov I. O., Bogdanov S. R. Statisticheskaia teoriia iavlenii perenosa v protsessakh khimicheskoi tekhnologii [Statistical Theory of Transfer Phenomena in the Processes of Chemical Technology]. Leningrad, Khimiya, 1983 (In Russian).

75. Melzak Z. A. A scalar transport equation, Trans. Amer. Math. Soc., 1957, vol. 85, pp. 547560. doi: 10.1090/S0002-9947-1957-0087880-6.

76. Higashitani K., Yamanchi K., Matsuno Y., Hosokawa G. Turbulent coagulation of particles dispersed in a viscous fluid, J. Chem. Eng. Japan, 1983, vol. 16, no. 4, pp. 299-304. doi: 10. 1252/jcej.16.299.

77. Kelbaliyev G. I., Rasulov S. R., Mustafayeva G. R. Modeling of phenomena of drop coalescence in oil emulsion breaking processes, Chem. Technol. Fuels Oils, 2018, vol. 54, no. 2, pp. 158-165. doi : 10.1007/s10553-018-0910-2.

78. Golovin A. M. On solving the equation of rain drop coagulation with allowance for condensation, Dokl. Akad. Nauk SSSR, 1963, vol.148, no.6, pp. 1290-1293 (In Russian).

79. Alopaeus V., Laakkonen M., Aittamaa J. Solution of population balances with breakage and agglomeration by high-order moment-conserving method of classes, Chem. Eng. Sci., 2006, vol. 61, no. 20, pp. 6732-6752. doi: 10.1016/j.ces.2006.07.010.

80. Maaß S., Wollny S., Sperling R., Kraume M. Numerical and experimental analysis of particle strain and breakage in turbulent dispersions, Chem. Eng. Res. Des., 2009, vol.87, no. 4, pp. 565-572. doi: 10.1016/j.cherd.2009.01.002.

81. Aristov S. N., Prosviryakov E. Yu. On one class of analytic solutions of the stationary axisymmetric convection Bénard-Marangoni viscous incompressible fluid, Vestn. Samar. Gos. Tekhn. Univ., Ser. Fiz.-Mat. Nauki [J. Samara State Tech. Univ., Ser. Phys. Math. Sci.], 2013, no. 3(32), pp. 110-118 (In Russian). doi: https://doi.org/10.14498/vsgtu1205.

82. Vlasova S. S., Prosviryakov E. Yu. Two-dimensional convection of an incompressible viscous fluid with the heat exchange on the free border, Vestn. Samar. Gos. Tekhn. Univ., Ser. Fiz.-Mat. Nauki [J. Samara State Tech. Univ., Ser. Phys. Math. Sci.], 2016, vol. 20, no. 3, pp. 567-577. doi: 10.14498/vsgtu1483.

83. Privalova V. V., Prosviryakov E. Yu. Couette-Hiemenz exact solutions for the steady creeping convective flow of a viscous incompressible fluid, with allowance made for heat recovery, Vestn. Samar. Gos. Tekhn. Univ., Ser. Fiz.-Mat. Nauki [J. Samara State Tech. Univ., Ser. Phys. Math. Sci.], 2018, vol. 22, no. 3, pp. 532-548. doi: 10.14498/vsgtu1638.

84. Sajjadi S., Zerfa M., Brooks B. W. Dynamic behaviour of drops in oil/water/oil dispersions, Chem. Eng. Sci., 2002, vol.57, no. 4, pp. 663-675. doi:10.1016/S0009-2509(01)00415-8.

85. Poston T., Stewart I. Catastrophe theory and its applications, Surveys and Reference Works in Mathematics, vol. 2. London, Pitman, 1978, xviii+491 pp.

86. Sevik M., Park S. H. The splitting of drops and bubbles by turbulent fluid flow, J. Fluids Eng., 1973, vol.95, no. 1, pp. 53-60. doi: 10.1115/1.3446958.

87. Risso F., Farbe J. Oscillations and breakup of a bubble immersed in a turbulent field, J. Fluid Mech., 1998, vol.372, pp. 323-335. doi: 10.1017/S0022112098002705. 
88. Kelbaliyev G. I., Suleimanov G. Z., Zorofi P. A., Gasanov A. A., Rustamova A. I. Extraction separation and cleaning of sewage waters by organic solvents with recirculation, Russ. J. Appl. Chem., 2011, vol. 84, no. 6, pp. 1114-1119. doi: 10.1134/S1070427211060383.

89. Sjöblom J., Urdahl O., Høiland H., Christy A. A., Johansen E. J. Water-in-crude oil emulsions. Formation, characterization, and destabilization, In: Surfactants and Macromolecules: Self-Assembly at Interfaces and in Bulk, Progress in Colloid and Polymer Science, 82. Darmstadt, Steinkopff, 1990, pp. 131-139. doi: 10.1007/BFb0118251.

90. Pozdnyshev G. N. Stabilizatsiia i razrushenie neftianykh emul'sii [Stabilization and destruction of oil emulsions]. Moscow, Nedra, 1982 (In Russian).

91. Tronov V. P. Razrushenie emul'sii pri dobyche nefti [The destruction of emulsions in oil production]. Moscow, Nedra, 1974 (In Russian). 